Table S1. Results of the GC-MS analysis of volatile components extracted by mechanical shock crushing from cordierite (from the central part of the crystal) from pegmatite of the Kuhilal deposit, Tajikistan.

\begin{tabular}{|c|c|c|c|c|c|}
\hline \multirow{2}{*}{ Formula } & \multirow{2}{*}{ Name } & \multirow{2}{*}{${ }^{1} \mathrm{CAS}$} & \multirow{2}{*}{${ }^{2} \mathrm{MW}$} & \multicolumn{2}{|c|}{ Cordierite-center } \\
\hline & & & & ${ }^{3} \mathrm{RT}, \min$ & ${ }^{4} \mathrm{~A}, \%$ \\
\hline \multicolumn{6}{|c|}{ Aliphatic hydrocarbons } \\
\hline \multicolumn{6}{|l|}{ Paraffins } \\
\hline $\mathrm{CH}_{4}$ & Methane & $74-82-8$ & 32 & 2.03 & 0.072 \\
\hline $\mathrm{C}_{2} \mathrm{H}_{6}$ & Ethane & $74-84-0$ & 30 & 2.78 & 0.012 \\
\hline $\mathrm{C}_{3} \mathrm{H}_{8}$ & n-Propane & $74-98-6$ & 44 & 4.68 & 0.001 \\
\hline $\mathrm{C}_{4} \mathrm{H}_{10}$ & Isobutane & $75-28-5$ & 58 & 6.44 & 0.018 \\
\hline $\mathrm{C}_{4} \mathrm{H}_{10}$ & n-Butane & $106-97-8$ & 58 & 7.22 & 0.051 \\
\hline $\mathrm{C}_{5} \mathrm{H}_{12}$ & n-Pentane & $109-66-0$ & 72 & 8.90 & 0.011 \\
\hline $\mathrm{C}_{6} \mathrm{H}_{14}$ & n-Hexane & $110-54-3$ & 86 & 12.32 & 0.024 \\
\hline $\mathrm{C}_{7} \mathrm{H}_{16}$ & n-Heptane & $142-82-5$ & 100 & 16.35 & 0.020 \\
\hline $\mathrm{C}_{8} \mathrm{H}_{18}$ & 2-Methylheptane & $592-27-8$ & 114 & 19.56 & 0.014 \\
\hline $\mathrm{C}_{8} \mathrm{H}_{18}$ & n-Octane & $111-65-9$ & 114 & 20.39 & 0.040 \\
\hline $\mathrm{C}_{9} \mathrm{H}_{20}$ & n-Nonane & $111-84-2$ & 128 & 24.25 & 0.058 \\
\hline $\mathrm{C}_{10} \mathrm{H}_{22}$ & n-Decane & $124-18-5$ & 142 & 27.82 & 0.013 \\
\hline $\mathrm{C}_{11} \mathrm{H}_{24}$ & n-Undecane & $1120-21-4$ & 156 & 31.11 & 0.012 \\
\hline $\mathrm{C}_{12} \mathrm{H}_{26}$ & n-Dodecane & $112-40-3$ & 170 & 34.25 & 0.008 \\
\hline $\mathrm{C}_{13} \mathrm{H}_{28}$ & n-Tridecane & $629-50-5$ & 184 & 38.28 & 0.038 \\
\hline $\mathrm{C}_{14} \mathrm{H}_{30}$ & n-Tetradecane & $629-59-4$ & 198 & 44.35 & 0.014 \\
\hline $\mathrm{C}_{15} \mathrm{H}_{32}$ & 5-Methyltetradecane & $25117-32-2$ & 212 & 46.27 & 0.021 \\
\hline $\mathrm{C}_{15} \mathrm{H}_{32}$ & n-Pentadecane & $629-62-9$ & 212 & 53.74 & 0.031 \\
\hline $\mathrm{C}_{16} \mathrm{H}_{34}$ & n-Hexadecane & $544-76-3$ & 226 & 68.65 & 0.032 \\
\hline $\mathrm{C}_{16} \mathrm{H}_{33} \mathrm{Cl}$ & 1-Chlorohexadecane & $4860-03-1$ & 240 & 120.33 & 0.026 \\
\hline \multicolumn{6}{|l|}{ Olefins } \\
\hline $\mathrm{C}_{2} \mathrm{H}_{4}$ & Ethylene & $74-85-1$ & 28 & 2.56 & 0.007 \\
\hline $\mathrm{C}_{2} \mathrm{H}_{2}$ & Acetylene & $74-86-2$ & 26 & 2.85 & 0.015 \\
\hline $\mathrm{C}_{3} \mathrm{H}_{6}$ & 1-Propene & $115-07-1$ & 42 & 4.33 & 0.020 \\
\hline $\mathrm{C}_{4} \mathrm{H}_{8}$ & 2-Methyl-1-propene & $115-11-7$ & 56 & 6.21 & 0.063 \\
\hline $\mathrm{C}_{5} \mathrm{H}_{8}$ & 1,4-Pentadiene & $591-93-5$ & 68 & 8.53 & 0.003 \\
\hline $\mathrm{C}_{5} \mathrm{H}_{10}$ & 1-Pentene & $109-67-1$ & 70 & 8.62 & 0.029 \\
\hline $\mathrm{C}_{5} \mathrm{H}_{8}$ & (E)-1,3-Pentadiene & $2004-70-8$ & 68 & 8.87 & 0.043 \\
\hline $\mathrm{C}_{5} \mathrm{H}_{8}$ & 3-Methyl-1,2-butadiene & $598-25-4$ & 68 & 9.15 & 0.001 \\
\hline $\mathrm{C}_{5} \mathrm{H}_{8}$ & 1,3-Pentadiene & $504-60-9$ & 68 & 9.38 & 0.001 \\
\hline $\mathrm{C}_{5} \mathrm{H}_{8}$ & (Z)-1,3-Pentadiene & $1574-41-0$ & 68 & 9.47 & $<0.001$ \\
\hline $\mathrm{C}_{6} \mathrm{H}$ & 1-Hexene & $592-41-6$ & 84 & 12.01 & 0.018 \\
\hline $\mathrm{C}_{6} \mathrm{H}_{10}$ & 4-Methyl-1,3-pentadiene & $926-56-7$ & 82 & 13.54 & 0.001 \\
\hline $\mathrm{C}_{6} \mathrm{H}_{10}$ & (Z)-3-Methyl-1,3-pentadiene & $2787-45-3$ & 82 & 13.70 & 0.001 \\
\hline $\mathrm{C}_{7} \mathrm{H}_{14}$ & 1-Heptene & $592-76-7$ & 98 & 16.00 & 0.018 \\
\hline $\mathrm{C}_{8} \mathrm{H}_{16}$ & 5-Methyl-3-heptene & $13172-91-3$ & 114 & 19.79 & 0.096 \\
\hline $\mathrm{C}_{8} \mathrm{H}_{16}$ & 2,5-Dimethyl-2-hexene & $3404-78-2$ & 112 & 20.01 & 0.057 \\
\hline $\mathrm{C}_{8} \mathrm{H}_{16}$ & (E)-4-Octene & $14850-23-8$ & 112 & 20.09 & 0.044 \\
\hline $\mathrm{C}_{8} \mathrm{H}_{16}$ & 2,3-Dimethyl-1-hexene & $16746-86-4$ & 112 & 20.21 & 0.034 \\
\hline $\mathrm{C}_{8} \mathrm{H}_{16}$ & (Z)-3-Octene & $14850-22-7$ & 112 & 20.29 & 0.005 \\
\hline $\mathrm{C}_{8} \mathrm{H}_{16}$ & (E)-2-Octene & $13389-42-9$ & 112 & 20.54 & 0.005 \\
\hline
\end{tabular}




\begin{tabular}{l|l}
$\mathrm{C}_{9} \mathrm{H}_{18}$ & 1-Nonene \\
$\mathrm{C}_{10} \mathrm{H}_{20}$ & 1-Decene \\
$\mathrm{C}_{11} \mathrm{H}_{22}$ & 1-Undecene \\
$\mathrm{C}_{12} \mathrm{H}_{24}$ & 1-Dodecene \\
$\mathrm{C}_{13} \mathrm{H}_{26}$ & 1-Tridecene \\
$\mathrm{C}_{14} \mathrm{H}_{28}$ & (E)-3-Tetradecene \\
$\mathrm{C}_{14} \mathrm{H}_{28}$ & 1-Tetradecene \\
$\mathrm{C}_{15} \mathrm{H}_{30}$ & 1-Pentadecene \\
$\mathrm{C}_{16} \mathrm{H}_{32}$ & 1-Hexadecene \\
$\mathrm{C}_{17} \mathrm{H}_{34}$ & 1-Heptadecene
\end{tabular}

\begin{tabular}{|l|c|c|c|}
$124-11-8$ & 126 & 23.98 & 0.018 \\
$872-05-9$ & 140 & 27.57 & 0.005 \\
$821-95-4$ & 154 & 30.91 & 0.009 \\
$112-41-4$ & 168 & 34.05 & 0.015 \\
$2437-56-1$ & 182 & 38.01 & 0.062 \\
$41446-68-8$ & 196 & 43.95 & 0.041 \\
$1120-36-1$ & 196 & 43.91 & 0.038 \\
$13360-61-7$ & 210 & 53.17 & 0.051 \\
$629-73-2$ & 224 & 73.44 & 0.017 \\
$6765-39-5$ & 238 & 115.09 & 0.043
\end{tabular}

Cyclic alkanes and alkenes

\begin{tabular}{l|l}
$\mathrm{C}_{6} \mathrm{H}_{10}$ & 4-Methylcyclopentene \\
$\mathrm{C}_{8} \mathrm{H}_{14}$ & 3-Propylcyclopentene
\end{tabular}

\section{Cyclic hydrocarbons}

Arenes

\begin{tabular}{l|l}
$\mathrm{C}_{6} \mathrm{H}_{6}$ & Benzene \\
$\mathrm{C}_{7} \mathrm{H}_{8}$ & Toluene \\
$\mathrm{C}_{8} \mathrm{H}_{10}$ & Ethylbenzene \\
$\mathrm{C}_{8} \mathrm{H}_{10}$ & p-Xylene \\
$\mathrm{C}_{8} \mathrm{H}_{8}$ & Styrene \\
$\mathrm{C}_{9} \mathrm{H}_{12}$ & Propylbenzene \\
$\mathrm{C}_{10} \mathrm{H}_{14}$ & o-Cymene \\
$\mathrm{C}_{10} \mathrm{H}_{14}$ & Butylbenzene \\
$\mathrm{C}_{11} \mathrm{H}_{16}$ & Pentylbenzene \\
$\mathrm{C}_{12} \mathrm{H}_{18}$ & Hexylbenzene \\
$\mathrm{C}_{13} \mathrm{H}_{20}$ & Heptylbenzene \\
$\mathrm{C}_{14} \mathrm{H}_{22}$ & Octylbenzene \\
$\mathrm{C}_{15} \mathrm{H}_{24}$ & Nonylbenzene
\end{tabular}

\begin{tabular}{|l|c|c|c|}
$1759-81-5$ & 82 & 12.56 & 0.016 \\
$34067-75-9$ & 110 & 21.39 & 0.001
\end{tabular}

71-43-2

108-88-3

$100-41-4$

$106-42-3$

$100-42-5$

103-65-1

527-84-4

104-51-8

538-68-1

1077-16-3

1078-71-3

2189-60-8

1081-77-2

78

92

106

106

104

120

134

134

148

162

176

190

204

13.01

17.43

0.019

21.44

0.017

0.035

21.70

0.004

22.37

0.002

25.23

0.002

28.04

0.004

28.93

0.002

32.34

0.012

35.75

0.003

38.89

0.016

46.24

0.008

Polycyclic aromatic hydrocarbons

71.24

0.012

\begin{tabular}{l|l}
$\mathrm{C}_{10} \mathrm{H}_{8}$ & Naphthalene \\
$\mathrm{C}_{11} \mathrm{H}_{10}$ & 2-Methylnaphthalene \\
$\mathrm{C}_{11} \mathrm{H}_{10}$ & 1-Methylnaphthalene
\end{tabular}

\begin{tabular}{|l}
$91-20-3$ \\
$91-57-6$ \\
$90-12-0$
\end{tabular}

128

142

32.67

35.77

0.001

142

36.27

$<0.001$

$<0.001$

Heterocyclic hydrocarbons

Dioxanes

\begin{tabular}{l|l}
$\mathrm{C}_{4} \mathrm{H}_{8} \mathrm{O}_{2}$ & 1,4-Dioxane \\
$\mathrm{C}_{4} \mathrm{H}_{8} \mathrm{O}_{2}$ & 1,3-Dioxane
\end{tabular}

Furans

\begin{tabular}{l|l}
$\mathrm{C}_{5} \mathrm{H}_{6} \mathrm{O}$ & 2-Methylfuran \\
$\mathrm{C}_{6} \mathrm{H}_{8} \mathrm{O}$ & 2-Ethylfuran \\
$\mathrm{C}_{7} \mathrm{H}_{10} \mathrm{O}$ & 2-Propylfuran \\
$\mathrm{C}_{8} \mathrm{H}_{12} \mathrm{O}$ & 2-Butylfuran \\
$\mathrm{C}_{9} \mathrm{H}_{14} \mathrm{O}$ & 2-Pentylfuran \\
$\mathrm{C}_{10} \mathrm{H}_{16} \mathrm{O}$ & 2-Hexylfuran \\
$\mathrm{C}_{11} \mathrm{H}_{18} \mathrm{O}$ & 2-Heptylfuran \\
$\mathrm{C}_{12} \mathrm{H}_{20} \mathrm{O}$ & 2-Octylfuran \\
$\mathrm{C}_{15} \mathrm{H}_{26} \mathrm{O}$ & 2-Decylfuran
\end{tabular}

123-91-1 505-22-6

88

88

15.15

17.89

$<0.001$

0.001

\begin{tabular}{|l|}
$534-22-5$ \\
$3208-16-0$ \\
$4229-91-8$ \\
$4466-24-4$ \\
$3777-69-3$ \\
$3777-70-6$ \\
$3777-71-7$ \\
$4179-38-8$ \\
$83469-85-6$
\end{tabular}

82

96

110

124

138

152

166

180

222
10.70

16.38

18.41

22.70

26.56

29.88

33.01

36.17

72.84
0.003

0.002

$<0.001$

0.002

$<0.001$

0.001

0.001

0.001

0.008 
Alcohols, ethers and esters

\begin{tabular}{|c|c|c|c|c|c|}
\hline $\mathrm{CH}_{4} \mathrm{O}$ & Methanol & $67-56-1$ & 32 & 4.83 & 0.181 \\
\hline $\mathrm{C}_{2} \mathrm{H}_{6} \mathrm{O}$ & Ethanol & $64-17-5$ & 46 & 6.81 & 0.040 \\
\hline $\mathrm{C}_{4} \mathrm{H}_{10} \mathrm{O}$ & 1-Butanol & $71-36-3$ & 74 & 13.19 & 0.041 \\
\hline $\mathrm{C}_{5} \mathrm{H}_{8} \mathrm{O}_{2}$ & Methyl methacrylate & $80-62-6$ & 100 & 14.85 & 0.027 \\
\hline $\mathrm{C}_{4} \mathrm{H}_{6} \mathrm{O}_{2}$ & $\gamma$-Butyrolactone & $96-48-0$ & 86 & 21.57 & 0.086 \\
\hline $\mathrm{C}_{6} \mathrm{H}_{10} \mathrm{O}$ & 2-Cyclohexen-1-ol & $822-67-3$ & 98 & 21.88 & 0.001 \\
\hline $\mathrm{C}_{5} \mathrm{H}_{8} \mathrm{O}_{2}$ & (E)-4-Cyclopentene-1,3-diol & $694-47-3$ & 100 & 23.15 & 0.014 \\
\hline $\mathrm{C}_{6} \mathrm{H}_{6} \mathrm{O}$ & Phenol & $108-95-2$ & 94 & 24.93 & 0.005 \\
\hline $\mathrm{C}_{8} \mathrm{H}_{18} \mathrm{O}$ & 2-Ethyl-1-hexanol & $104-76-7$ & 130 & 28.12 & 0.054 \\
\hline $\mathrm{C}_{6} \mathrm{H}_{10} \mathrm{O}_{2}$ & $\gamma$-Caprolactone & $695-06-7$ & 114 & 29.73 & 0.001 \\
\hline $\mathrm{C}_{8} \mathrm{H}_{10} \mathrm{O}_{2}$ & 2-Phenoxyethanol & $122-99-6$ & 138 & 32.87 & 0.016 \\
\hline $\mathrm{C}_{8} \mathrm{H}_{14} \mathrm{O}_{2}$ & $\gamma$-Octalactone & $104-50-7$ & 142 & 34.90 & 0.005 \\
\hline $\mathrm{C}_{10} \mathrm{H}_{20} \mathrm{O}$ & (E)-2-Decen-1-ol & $18409-18-2$ & 156 & 37.05 & 0.018 \\
\hline $\mathrm{C}_{9} \mathrm{H}_{16} \mathrm{O}_{2}$ & $\gamma$-Nonalactone & $104-61-0$ & 156 & 39.44 & 0.013 \\
\hline $\mathrm{C}_{12} \mathrm{H}_{22} \mathrm{O}_{2}$ & $\gamma$-Dodecalactone & $2305-05-7$ & 198 & 85.33 & 0.007 \\
\hline $\mathrm{C}_{14} \mathrm{H}_{18} \mathrm{O}_{4}$ & Dipropyl phthalate & $131-16-8$ & 250 & 126.73 & 0.089 \\
\hline \multicolumn{6}{|l|}{ Aldehydes } \\
\hline $\mathrm{C}_{2} \mathrm{H}_{4} \mathrm{O}$ & Acetaldehyde & $75-07-0$ & 44 & 5.34 & 0.350 \\
\hline $\mathrm{C}_{3} \mathrm{H}_{4} \mathrm{O}$ & 2-Propenal & $107-02-8$ & 56 & 7.62 & 0.019 \\
\hline $\mathrm{C}_{3} \mathrm{H}_{6} \mathrm{O}$ & n-Propanal & $123-38-6$ & 58 & 7.82 & 0.068 \\
\hline $\mathrm{C}_{3} \mathrm{H}_{4} \mathrm{O}_{2}$ & 2-Oxopropanal & $78-98-8$ & 72 & 8.25 & 0.043 \\
\hline $\mathrm{C}_{4} \mathrm{H}_{6} \mathrm{O}$ & 2-Methylpropanal & $78-84-2$ & 70 & 10.11 & 0.090 \\
\hline $\mathrm{C}_{4} \mathrm{H}_{8} \mathrm{O}$ & n-Butanal & $123-72-8$ & 72 & 10.95 & 0.009 \\
\hline $\mathrm{C}_{5} \mathrm{H}_{10} \mathrm{O}$ & 3-Methylbutanal & $590-86-3$ & 86 & 14.07 & 0.028 \\
\hline $\mathrm{C}_{5} \mathrm{H}_{10} \mathrm{O}$ & n-Pentanal & $110-62-3$ & 86 & 15.07 & 0.041 \\
\hline $\mathrm{C}_{5} \mathrm{H}_{4} \mathrm{O}_{2}$ & Furfural & $98-01-1$ & 96 & 17.98 & 0.001 \\
\hline $\mathrm{C}_{5} \mathrm{H}_{4} \mathrm{O}_{2}$ & 3-Furaldehyde & $498-60-2$ & 96 & 18.88 & 0.010 \\
\hline $\mathrm{C}_{6} \mathrm{H}_{12} \mathrm{O}$ & n-Hexanal & $66-25-1$ & 100 & 19.36 & 0.152 \\
\hline $\mathrm{C}_{6} \mathrm{H}_{6} \mathrm{O}_{2}$ & $\begin{array}{l}\text { 5-Methyl-2- } \\
\text { furancarboxaldehyde }\end{array}$ & $620-02-0$ & 110 & 23.88 & 0.003 \\
\hline $\mathrm{C}_{7} \mathrm{H}_{14} \mathrm{O}$ & n-Heptanal & $111-71-7$ & 114 & 23.43 & 0.156 \\
\hline $\mathrm{C}_{7} \mathrm{H}_{6} \mathrm{O}$ & Benzaldehyde & $100-52-7$ & 106 & 24.59 & 0.020 \\
\hline $\mathrm{C}_{8} \mathrm{H}_{16} \mathrm{O}$ & 2-Ethylhexanal & $123-05-7$ & 128 & 25.86 & 0.017 \\
\hline $\mathrm{C}_{8} \mathrm{H}_{16} \mathrm{O}$ & n-Octanal & $124-13-0$ & 128 & 27.19 & 0.244 \\
\hline $\mathrm{C}_{9} \mathrm{H}_{18} \mathrm{O}$ & n-Nonanal & $124-19-6$ & 142 & 30.65 & 0.300 \\
\hline $\mathrm{C}_{6} \mathrm{H}_{6} \mathrm{O}_{3}$ & 5-Hydroxymethylfurfural & $67-47-0$ & 126 & 31.39 & 0.021 \\
\hline $\mathrm{C}_{10} \mathrm{H}_{20} \mathrm{O}$ & n-Decanal & $112-31-2$ & 156 & 35.87 & 0.373 \\
\hline $\mathrm{C}_{11} \mathrm{H}_{22} \mathrm{O}$ & n-Undecanal & $112-44-7$ & 170 & 37.81 & 0.117 \\
\hline $\mathrm{C}_{12} \mathrm{H}_{24} \mathrm{O}$ & n-Dodecanal & $112-54-9$ & 184 & 43.68 & 0.071 \\
\hline $\mathrm{C}_{13} \mathrm{H}_{26} \mathrm{O}$ & n-Tridecanal & $10486-19-8$ & 198 & 52.77 & 0.025 \\
\hline $\mathrm{C}_{14} \mathrm{H}_{28} \mathrm{O}$ & n-Tetradecanal & $124-25-4$ & 212 & 67.02 & 0.014 \\
\hline $\mathrm{C}_{15} \mathrm{H}_{30} \mathrm{O}$ & n-Pentadecanal & $2765-11-9$ & 226 & 89.93 & 0.050 \\
\hline \multicolumn{6}{|l|}{ Ketones } \\
\hline $\mathrm{C}_{3} \mathrm{H}_{6} \mathrm{O}$ & Acetone & $67-64-1$ & 58 & 7.99 & 0.017 \\
\hline $\mathrm{C}_{4} \mathrm{H}_{6} \mathrm{O}$ & 3-Buten-2-one & $78-94-4$ & 70 & 10.76 & 0.002 \\
\hline $\mathrm{C}_{4} \mathrm{H}_{6} \mathrm{O} 2$ & 2,3-Butanedione & $431-03-8$ & 86 & 10.99 & 0.001 \\
\hline $\mathrm{C}_{4} \mathrm{H}_{8} \mathrm{O}$ & 2-Butanone & $78-93-3$ & 72 & 11.13 & 0.005 \\
\hline
\end{tabular}




\begin{tabular}{l|l}
$\mathrm{C}_{5} \mathrm{H}_{10} \mathrm{O}$ & 2-Pentanone \\
$\mathrm{C}_{5} \mathrm{H}_{8} \mathrm{O}$ & Cyclopentanone \\
$\mathrm{C}_{6} \mathrm{H}_{12} \mathrm{O}$ & 2-Hexanone \\
$\mathrm{C}_{7} \mathrm{H}_{14} \mathrm{O}$ & 2-Heptanone \\
$\mathrm{C}_{8} \mathrm{H}_{16} \mathrm{O}$ & 6-Methyl-2-heptanone \\
$\mathrm{C}_{8} \mathrm{H}_{14} \mathrm{O}$ & 6-Methyl-5-hepten-2-one \\
$\mathrm{C}_{8} \mathrm{H}_{16} \mathrm{O}$ & 2-Octanone \\
$\mathrm{C}_{9} \mathrm{H}_{18} \mathrm{O}$ & 2-Nonanone \\
$\mathrm{C}_{10} \mathrm{H}_{20} \mathrm{O}$ & 2-Decanone \\
$\mathrm{C}_{11} \mathrm{H}_{22} \mathrm{O}$ & 2-Undecanone \\
$\mathrm{C}_{12} \mathrm{H}_{24} \mathrm{O}$ & 2-Dodecanone \\
$\mathrm{C}_{13} \mathrm{H}_{26} \mathrm{O}$ & 2-Tridecanone \\
$\mathrm{C}_{14} \mathrm{H}_{28} \mathrm{O}$ & 2-Tetradecanone \\
$\mathrm{C}_{15} \mathrm{H}_{30} \mathrm{O}$ & 2-Pentadecanone
\end{tabular}

\begin{tabular}{|l|}
$107-87-9$ \\
$120-92-3$ \\
$591-78-6$ \\
$110-43-0$ \\
$928-68-7$ \\
$110-93-0$ \\
$111-13-7$ \\
$821-55-6$ \\
$693-54-9$ \\
$112-12-9$ \\
$6175-49-1$ \\
$593-08-8$ \\
$2345-27-9$ \\
$2345-28-0$
\end{tabular}

\begin{tabular}{c|c|c}
86 & 14.84 & 0.010 \\
84 & 17.63 & 0.002 \\
100 & 19.09 & 0.011 \\
114 & 23.11 & 0.010 \\
128 & 26.07 & 0.013 \\
126 & 26.36 & 0.009 \\
128 & 27.07 & 0.013 \\
142 & 30.33 & 0.012 \\
156 & 33.49 & 0.037 \\
170 & 37.28 & 0.032 \\
184 & 42.87 & 0.014 \\
198 & 51.44 & 0.049 \\
212 & 64.89 & 0.011 \\
226 & 86.38 & 0.071
\end{tabular}

Carboxylic acids

\begin{tabular}{l|l|l|l|l|l}
$\mathrm{C}_{2} \mathrm{H}_{4} \mathrm{O}_{2}$ & Acetic acid & $64-19-7$ & 60 & 11.48 & 0.095 \\
$\mathrm{C}_{3} \mathrm{H}_{6} \mathrm{O}_{2}$ & n-Propanoic acid & $79-09-4$ & 74 & 15.98 & 0.007 \\
$\mathrm{C}_{4} \mathrm{H}_{8} \mathrm{O}_{2}$ & n-Butanoic acid & $107-92-6$ & 88 & 19.34 & 0.033 \\
$\mathrm{C}_{5} \mathrm{H}_{10} \mathrm{O}_{2}$ & 3-Methylbutanoic acid & $503-74-2$ & 102 & 22.35 & 0.004 \\
$\mathrm{C}_{5} \mathrm{H}_{10} \mathrm{O}_{2}$ & n-Pentanoic acid & $109-52-4$ & 102 & 23.26 & 0.021 \\
$\mathrm{C}_{6} \mathrm{H}_{12} \mathrm{O}_{2}$ & n-Hexanoic acid & $142-62-1$ & 116 & 26.69 & 0.040 \\
$\mathrm{C}_{7} \mathrm{H}_{14} \mathrm{O}_{2}$ & n-Heptanoic acid & $111-14-8$ & 130 & 30.11 & 0.024 \\
$\mathrm{C}_{8} \mathrm{H}_{16} \mathrm{O}_{2}$ & n-Octanoic acid & $124-07-2$ & 144 & 33.12 & 0.034 \\
$\mathrm{C}_{9} \mathrm{H}_{18} \mathrm{O}_{2}$ & n-Nonanoic acid & $112-05-0$ & 158 & 36.61 & 0.054 \\
$\mathrm{C}_{10} \mathrm{H}_{20} \mathrm{O}_{2}$ & n-Decanoic acid & $334-48-5$ & 172 & 41.39 & 0.046 \\
$\mathrm{C}_{11} \mathrm{H}_{22} \mathrm{O}_{2}$ & n-Undecanoic acid & $112-37-8$ & 186 & 63.08 & 0.014 \\
$\mathrm{C}_{11} \mathrm{H}_{14} \mathrm{O}_{3}$ & 2,4,6-Trimethylmandelic acid & $20797-56-2$ & 194 & 87.24 & 0.001 \\
$\mathrm{C}_{12} \mathrm{H}_{24} \mathrm{O}_{2}$ & n-Dodecanoic acid & $143-07-7$ & 200 & 73.89 & 0.056 \\
$\mathrm{C}_{13} \mathrm{H}_{26} \mathrm{O}_{2}$ & n-Tridecanoic acid & $638-53-9$ & 214 & 113.49 & 0.140
\end{tabular}

\begin{tabular}{l|l}
$\mathrm{H}_{2} \mathrm{~S}$ & Hydrogen sulfide \\
$\mathrm{COS}$ & Carbonyl sulfide \\
$\mathrm{O}_{2} \mathrm{~S}$ & Sulfur dioxide \\
$\mathrm{CS}_{2}$ & Carbon disulfide \\
$\mathrm{C}_{2} \mathrm{H}_{6} \mathrm{~S}$ & Dimethyl sulfide \\
$\mathrm{C}_{2} \mathrm{H}_{6} \mathrm{~S}_{2}$ & Dimethyl disulfide \\
$\mathrm{C}_{10} \mathrm{H}_{16} \mathrm{~S}$ & 2-Hexylthiophene
\end{tabular}

Sulfonated compounds

\begin{tabular}{|l|c|c|c|}
$7783-06-4$ & 34 & 3.43 & $<0.001$ \\
$463-58-1$ & 60 & 3.78 & 0.002 \\
$7446-09-5$ & 64 & 5.16 & 0.009 \\
$75-15-0$ & 76 & 8.30 & 0.003 \\
$75-18-3$ & 62 & 8.19 & 0.006 \\
$624-92-0$ & 94 & 15.83 & 0.002 \\
$18794-77-9$ & 168 & 35.30 & $<0.001$
\end{tabular}

\begin{tabular}{l|l}
$\mathrm{N}_{2}$ & Nitrogen \\
$\mathrm{C}_{5} \mathrm{H}_{9} \mathrm{~N}$ & 1-Isocyanobutane \\
$\mathrm{CH}_{3} \mathrm{NO}$ & Formamide \\
$\mathrm{C}_{5} \mathrm{H}_{5} \mathrm{~N}$ & Pyridine \\
$\mathrm{C}_{6} \mathrm{H}_{11} \mathrm{NO}$ & 6-Methyl-2-piperidinone \\
$\mathrm{C}_{4} \mathrm{H}_{5} \mathrm{NO}_{2}$ & Succinimide
\end{tabular}

\begin{tabular}{|l|}
$7727-37-9$ \\
$2769-64-4$ \\
$75-12-7$ \\
$110-86-1$ \\
$4775-98-8$ \\
$123-56-8$
\end{tabular}

\begin{tabular}{c|c|c}
28 & 1.98 & 0.072 \\
83 & 6.56 & 0.015 \\
45 & 8.37 & 0.001 \\
79 & 16.10 & 0.006 \\
113 & 22.22 & 0.001 \\
99 & 29.32 & 0.023
\end{tabular}

Oxides

Inorganic compounds

$\mathrm{H}_{2} \mathrm{O} \quad$ Water

| 7732-18-5 
Note: ${ }^{1} \mathrm{CAS}$ - unique numerical identifier of chemical compounds included in the register Chemical Abstracts Service (https://www.cas.org); ${ }^{2} \mathrm{MW}$ - nominal mass; ${ }^{3} \mathrm{RT}$ - retention time; ${ }^{4} \mathrm{~A}$ - normalized area (the area ratio of the individual gas mixture components to the summ of the areas of all the components in the chromatogram). 
Table S2. Results of the GC-MS analysis of volatile components extracted by mechanical shock crushing from cordierite (from the edge zone of the crystal) from pegmatite of the Kukhilal deposit, Tajikistan.

\begin{tabular}{|c|c|c|c|c|c|}
\hline \multirow{2}{*}{ Formula } & \multirow{2}{*}{ Name } & \multirow{2}{*}{${ }^{1} \mathrm{CAS}$} & \multirow{2}{*}{${ }^{2} \mathrm{MW}$} & \multicolumn{2}{|c|}{ Cordierite-edge } \\
\hline & & & & ${ }^{3} \mathrm{RT}$, min & ${ }^{4} \mathrm{~A}, \%$ \\
\hline \multicolumn{6}{|c|}{ Aliphatic hydrocarbons } \\
\hline \multicolumn{6}{|l|}{ Paraffins } \\
\hline $\mathrm{CH}_{4}$ & Methane & $74-82-8$ & 32 & 2.02 & 0.058 \\
\hline $\mathrm{C}_{2} \mathrm{H}_{6}$ & Ethane & 74-84-0 & 30 & 2.85 & 0.034 \\
\hline $\mathrm{C}_{3} \mathrm{H}_{8}$ & n-Propane & $74-98-6$ & 44 & 4.49 & 0.079 \\
\hline $\mathrm{C}_{4} \mathrm{H}_{10}$ & Isobutane & $75-28-5$ & 58 & 6.45 & 0.036 \\
\hline $\mathrm{C}_{4} \mathrm{H}_{10}$ & n-Butane & $106-97-8$ & 58 & 7.22 & 0.047 \\
\hline $\mathrm{C}_{5} \mathrm{H}_{12}$ & n-Pentane & $109-66-0$ & 72 & 8.92 & 0.024 \\
\hline $\mathrm{C}_{6} \mathrm{H}_{14}$ & n-Hexane & $110-54-3$ & 86 & 12.36 & 0.071 \\
\hline $\mathrm{C}_{7} \mathrm{H}_{16}$ & n-Heptane & $142-82-5$ & 100 & 16.36 & 0.036 \\
\hline $\mathrm{C}_{8} \mathrm{H}_{18}$ & 2-Methylheptane & $592-27-8$ & 114 & 19.57 & 0.059 \\
\hline $\mathrm{C}_{8} \mathrm{H}_{16}$ & 3-Methyl-4-methylenehexane & $3404-67-9$ & 112 & 19.66 & 0.091 \\
\hline $\mathrm{C}_{8} \mathrm{H}_{16}$ & 2,5-Dimethylhexane & $592-13-2$ & 114 & 19.80 & 0.259 \\
\hline $\mathrm{C}_{8} \mathrm{H}_{18}$ & n-Octane & $111-65-9$ & 114 & 20.39 & 0.116 \\
\hline $\mathrm{C}_{9} \mathrm{H}_{20}$ & n-Nonane & $111-84-2$ & 128 & 24.24 & 0.087 \\
\hline $\mathrm{C}_{10} \mathrm{H}_{22}$ & n-Decane & $124-18-5$ & 142 & 27.82 & 0.047 \\
\hline $\mathrm{C}_{11} \mathrm{H}_{24}$ & n-Undecane & $1120-21-4$ & 156 & 31.13 & 0.027 \\
\hline $\mathrm{C}_{12} \mathrm{H}^{26}$ & n-Dodecane & $112-40-3$ & 170 & 34.25 & 0.031 \\
\hline $\mathrm{C}_{13} \mathrm{H}_{28}$ & $\mathrm{n}$-Tridecane & $629-50-5$ & 184 & 38.28 & 0.110 \\
\hline $\mathrm{C}_{14} \mathrm{H}_{30}$ & n-Tetradecane & $629-59-4$ & 198 & 44.40 & 0.043 \\
\hline $\mathrm{C}_{15} \mathrm{H}_{32}$ & 5-Methyltetradecane & $25117-32-2$ & 212 & 46.23 & 0.026 \\
\hline $\mathrm{C}_{15} \mathrm{H}_{32}$ & n-Pentadecane & $629-62-9$ & 212 & 53.81 & 0.084 \\
\hline $\mathrm{C}_{16} \mathrm{H}_{34}$ & n-Hexadecane & $544-76-3$ & 226 & 68.79 & 0.057 \\
\hline $\mathrm{C}_{19} \mathrm{H}_{40}$ & $\begin{array}{l}2,6,10,14- \\
\text { Tetramethylpentadecane }\end{array}$ & $1921-70-6$ & 268 & 92.81 & 0.111 \\
\hline $\mathrm{C}_{16} \mathrm{H}_{33} \mathrm{Cl}$ & 1-Chlorohexadecane & 4860-03-1 & 240 & 120.69 & 0.062 \\
\hline \multicolumn{6}{|l|}{ Olefins } \\
\hline $\mathrm{C}_{2} \mathrm{H}_{4}$ & Ethylene & $74-85-1$ & 28 & 2.60 & 0.016 \\
\hline $\mathrm{C}_{3} \mathrm{H}_{6}$ & 1-Propene & $115-07-1$ & 42 & 4.36 & 0.077 \\
\hline $\mathrm{C}_{4} \mathrm{H}_{8}$ & 2-Methyl-1-propene & $115-11-7$ & 56 & 6.22 & 0.158 \\
\hline $\mathrm{C}_{5} \mathrm{H}_{8}$ & 1,4-Pentadiene & $591-93-5$ & 68 & 8.52 & 0.004 \\
\hline $\mathrm{C}_{5} \mathrm{H}_{10}$ & 1-Pentene & $109-67-1$ & 70 & 8.63 & 0.045 \\
\hline $\mathrm{C}_{5} \mathrm{H}_{8}$ & (E)-1,3-Pentadiene & $2004-70-8$ & 68 & 8.88 & 0.058 \\
\hline $\mathrm{C}_{5} \mathrm{H}_{8}$ & 3-Methyl-1,2-butadiene & $598-25-4$ & 68 & 9.16 & 0.003 \\
\hline $\mathrm{C}_{5} \mathrm{H}_{8}$ & 1,3-Pentadiene & $504-60-9$ & 68 & 9.40 & 0.003 \\
\hline $\mathrm{C}_{5} \mathrm{H}_{8}$ & (Z)-1,3-Pentadiene & $1574-41-0$ & 68 & 9.50 & 0.001 \\
\hline $\mathrm{C}_{6} \mathrm{H}_{12}$ & 1-Hexene & $592-41-6$ & 84 & 12.02 & 0.041 \\
\hline $\mathrm{C}_{6} \mathrm{H}_{10}$ & 4-Methyl-1,3-pentadiene & $926-56-7$ & 82 & 13.55 & 0.004 \\
\hline $\mathrm{C}_{6} \mathrm{H}_{10}$ & (Z)-3-Methyl-1,3-pentadiene & $2787-45-3$ & 82 & 13.74 & 0.004 \\
\hline $\mathrm{C}_{7} \mathrm{H}_{14}$ & 1-Heptene & $592-76-7$ & 98 & 16.01 & 0.040 \\
\hline $\mathrm{C}_{8} \mathrm{H}_{16}$ & 2,5-Dimethyl-2-hexene & $3404-78-2$ & 112 & 20.00 & 0.268 \\
\hline $\mathrm{C}_{8} \mathrm{H}_{16}$ & (E)-3-Octene & $14919-01-8$ & 112 & 20.20 & 0.080 \\
\hline $\mathrm{C}_{8} \mathrm{H}_{16}$ & (Z)-3-Octene & $14850-22-7$ & 112 & 20.29 & 0.128 \\
\hline
\end{tabular}




\begin{tabular}{l|l}
$\mathrm{C}_{8} \mathrm{H}_{16}$ & 2-Octene \\
$\mathrm{C}_{9} \mathrm{H}_{18}$ & 1-Nonene \\
$\mathrm{C}_{10} \mathrm{H}_{20}$ & 1-Decene \\
$\mathrm{C}_{11} \mathrm{H}_{22}$ & 1-Undecene \\
$\mathrm{C}_{12} \mathrm{H}_{24}$ & 1-Dodecene \\
$\mathrm{C}_{13} \mathrm{H}_{26}$ & 1-Tridecene \\
$\mathrm{C}_{14} \mathrm{H}_{28}$ & (E)-3-Tetradecene \\
$\mathrm{C}_{14} \mathrm{H}_{28}$ & 1-Tetradecene \\
$\mathrm{C}_{15} \mathrm{H}_{30}$ & 1-Pentadecene \\
$\mathrm{C}_{16} \mathrm{H}_{32}$ & 1-Hexadecene \\
$\mathrm{C}_{17} \mathrm{H}_{34}$ & 1-Heptadecene
\end{tabular}

Cyclic alkanes and alkenes

\begin{tabular}{l|l}
$\mathrm{C}_{6} \mathrm{H}_{10}$ & 4-Methylcyclopentene \\
$\mathrm{C}_{8} \mathrm{H}_{14}$ & 3-Propylcyclopentene
\end{tabular}

Arenes

$\mathrm{C}_{6} \mathrm{H}_{6}$

$\mathrm{C}_{7} \mathrm{H}_{8}$

$\mathrm{C}_{8} \mathrm{H}_{10}$

$\mathrm{C}_{8} \mathrm{H}_{10}$

$\mathrm{C}_{8} \mathrm{H}_{8}$

$\mathrm{C}_{9} \mathrm{H}_{12}$

$\mathrm{C}_{10} \mathrm{H}_{14}$

$\mathrm{C}_{10} \mathrm{H}_{14}$

$\mathrm{C}_{11} \mathrm{H}_{16}$

$\mathrm{C}_{12} \mathrm{H}_{18}$

$\mathrm{C}_{13} \mathrm{H}_{20}$

$\mathrm{C}_{14} \mathrm{H}_{22}$

\begin{tabular}{l|l}
$\mathrm{C}_{15} \mathrm{H}_{24}$ & Nonylbenzene \\
Polycyclic aromatic hydrocarbons
\end{tabular}

\begin{tabular}{l|l}
$\mathrm{C}_{10} \mathrm{H}_{8}$ & Naphthalene \\
$\mathrm{C}_{11} \mathrm{H}_{10}$ & 2-Methylnaphthalene \\
$\mathrm{C}_{11} \mathrm{H}_{10}$ & 1-Methylnaphthalene
\end{tabular}

\begin{tabular}{|l|c|c|c|}
$111-67-1$ & 112 & 20.54 & 0.083 \\
$124-11-8$ & 126 & 24.01 & 0.157 \\
$872-05-9$ & 140 & 27.59 & 0.010 \\
$821-95-4$ & 154 & 30.93 & 0.005 \\
$112-41-4$ & 168 & 34.07 & 0.069 \\
$2437-56-1$ & 182 & 38.06 & 0.166 \\
$41446-68-8$ & 196 & 43.95 & 0.077 \\
$1120-36-1$ & 196 & 44.03 & 0.077 \\
$13360-61-7$ & 210 & 53.27 & 0.129 \\
$629-73-2$ & 224 & 73.54 & 0.026 \\
$6765-39-5$ & 238 & 116.30 & 0.100
\end{tabular}

Cyclic hydrocarbons

\begin{tabular}{|c|c|c|c|}
\hline $1759-81-5$ & 82 & 12.57 & 0.034 \\
\hline $34067-75-9$ & 110 & 21.40 & 0.004 \\
\hline $71-43-2$ & 78 & 13.02 & 0.043 \\
\hline $108-88-3$ & 92 & 17.46 & 0.042 \\
\hline $100-41-4$ & 106 & 21.45 & 0.002 \\
\hline $106-42-3$ & 106 & 21.72 & 0.008 \\
\hline $100-42-5$ & 104 & 22.38 & 0.007 \\
\hline $103-65-1$ & 120 & 25.26 & 0.003 \\
\hline $527-84-4$ & 134 & 28.03 & 0.019 \\
\hline $104-51-8$ & 134 & 28.95 & 0.004 \\
\hline 538-68-1 & 148 & 32.31 & 0.013 \\
\hline $1077-16-3$ & 162 & 35.78 & 0.008 \\
\hline $1078-71-3$ & 176 & 38.86 & 0.033 \\
\hline 2189-60-8 & 190 & 44.96 & 0.018 \\
\hline $1081-77-2$ & 204 & 71.16 & 0.024 \\
\hline $91-20-3$ & 128 & 32.64 & 0.004 \\
\hline $91-57-6$ & 142 & 35.28 & 0.003 \\
\hline $90-12-0$ & 142 & 36.15 & 0.002 \\
\hline
\end{tabular}

Heterocyclic hydrocarbons

Dioxanes

\begin{tabular}{l|l}
$\mathrm{C}_{4} \mathrm{H}_{8} \mathrm{O}_{2}$ & 1,4-Dioxane \\
$\mathrm{C}_{4} \mathrm{H}_{8} \mathrm{O}_{2}$ & 1,3-Dioxane
\end{tabular}

$\mid$\begin{tabular}{|l}
$123-91-1$ \\
$505-22-6$
\end{tabular}

88

88

14.17

0.001

17.91

0.001

Furans

\begin{tabular}{l|l}
$\mathrm{C}_{5} \mathrm{H}_{6} \mathrm{O}$ & 2-Methylfuran \\
$\mathrm{C}_{6} \mathrm{H}_{8} \mathrm{O}$ & 2-Ethylfuran \\
$\mathrm{C}_{7} \mathrm{H}_{10} \mathrm{O}$ & 2-Propylfuran \\
$\mathrm{C}_{8} \mathrm{H}_{12} \mathrm{O}$ & 2-Butylfuran \\
$\mathrm{C}_{9} \mathrm{H}_{14} \mathrm{O}$ & 2-Pentylfuran \\
$\mathrm{C}_{10} \mathrm{H}_{16} \mathrm{O}$ & 2-Hexylfuran \\
$\mathrm{C}_{11} \mathrm{H}_{18} \mathrm{O}$ & 2-Heptylfuran \\
$\mathrm{C}_{12} \mathrm{H}_{20} \mathrm{O}$ & 2-Octylfuran \\
$\mathrm{C}_{15} \mathrm{H}_{26} \mathrm{O}$ & 2-Decylfuran
\end{tabular}

\begin{tabular}{l|}
$534-22-5$ \\
$3208-16-0$ \\
$4229-91-8$ \\
$4466-24-4$ \\
$3777-69-3$ \\
$3777-70-6$ \\
$3777-71-7$ \\
$4179-38-8$ \\
$83469-85-6$
\end{tabular}

82
96
110
124
138
152
166
180
222

10.69

0.005

0.006

0.001

0.003

0.018

26.55

0.003

0.001

33.00

0.001

36.16

72.37 
Alcohols, ethers and esters

\section{Oxygenated hydrocarbons}

\begin{tabular}{|c|c|c|c|c|}
\hline $\mathrm{CH}_{4} \mathrm{O}$ & Methanol & $67-56-1$ & 32 & 4.54 \\
\hline $\mathrm{C}_{2} \mathrm{H}_{6} \mathrm{O}$ & Ethanol & $64-17-5$ & 46 & 6.67 \\
\hline $\mathrm{C}_{4} \mathrm{H}_{10} \mathrm{O}$ & 1-Butanol & $71-36-3$ & 74 & 13.19 \\
\hline $\mathrm{C}_{5} \mathrm{H}_{8} \mathrm{O}_{2}$ & Methyl methacrylate & $80-62-6$ & 100 & 14.87 \\
\hline $\mathrm{C}_{4} \mathrm{H}_{6} \mathrm{O}_{2}$ & $\gamma$-Butyrolactone & $96-48-0$ & 86 & 21.57 \\
\hline $\mathrm{C}_{6} \mathrm{H}_{6} \mathrm{O}$ & Phenol & $108-95-2$ & 94 & 24.96 \\
\hline $\mathrm{C}_{5} \mathrm{H}_{8} \mathrm{O}_{2}$ & $\delta$-Valerolactone & $542-28-9$ & 100 & 26.39 \\
\hline $\mathrm{C}_{8} \mathrm{H}_{18} \mathrm{O}$ & 2-Ethyl-1-hexanol & $104-76-7$ & 130 & 28.17 \\
\hline $\mathrm{C}_{6} \mathrm{H}_{10} \mathrm{O}_{2}$ & $\gamma$-Caprolactone & $695-06-7$ & 114 & 29.75 \\
\hline $\mathrm{C}_{5} \mathrm{H}_{8} \mathrm{Cl}_{2} \mathrm{O}_{2}$ & $\begin{array}{l}\text { Methyl ester 3,4- } \\
\text { dichlorobutanoic acid }\end{array}$ & $819-93-2$ & 170 & 30.10 \\
\hline $\mathrm{C}_{8} \mathrm{H}_{10} \mathrm{O}_{2}$ & 2-Phenoxyethanol & $122-99-6$ & 138 & 32.91 \\
\hline $\mathrm{C}_{8} \mathrm{H}_{14} \mathrm{O}_{2}$ & $\gamma$-Octalactone & $104-50-7$ & 142 & 34.93 \\
\hline $\mathrm{C}_{10} \mathrm{H}_{20} \mathrm{O}$ & (E)-2-Decen-1-ol & $18409-18-2$ & 156 & 36.95 \\
\hline $\mathrm{C}_{9} \mathrm{H}_{16} \mathrm{O}_{2}$ & $\gamma$-Nonalactone & 104-61-0 & 156 & 39.49 \\
\hline $\mathrm{C}_{12} \mathrm{H}_{22} \mathrm{O}_{2}$ & $\gamma$-Dodecalactone & $2305-05-7$ & 198 & 86.51 \\
\hline $\mathrm{C}_{14} \mathrm{H}_{18} \mathrm{O}_{4}$ & Dipropyl phthalate & $131-16-8$ & 250 & 127.04 \\
\hline \multicolumn{5}{|l|}{ Aldehydes } \\
\hline $\mathrm{C}_{2} \mathrm{H}_{4} \mathrm{O}$ & Acetaldehyde & $75-07-0$ & 44 & 5.09 \\
\hline $\mathrm{C}_{3} \mathrm{H}_{4} \mathrm{O}$ & 2-Propenal & $107-02-8$ & 56 & 7.59 \\
\hline $\mathrm{C}_{3} \mathrm{H}_{6} \mathrm{O}$ & n-Propanal & $123-38-6$ & 58 & 7.95 \\
\hline $\mathrm{C}_{3} \mathrm{H}_{4} \mathrm{O}_{2}$ & 2-Oxopropanal & $78-98-8$ & 72 & 8.27 \\
\hline $\mathrm{C}_{4} \mathrm{H}_{6} \mathrm{O}$ & 2-Methylpropanal & 78-84-2 & 70 & 10.13 \\
\hline $\mathrm{C}_{4} \mathrm{H}_{8} \mathrm{O}$ & n-Butanal & $123-72-8$ & 72 & 10.98 \\
\hline $\mathrm{C}_{5} \mathrm{H}_{10} \mathrm{O}$ & 3-Methylbutanal & $590-86-3$ & 86 & 14.09 \\
\hline $\mathrm{C}_{5} \mathrm{H}_{10} \mathrm{O}$ & n-Pentanal & $110-62-3$ & 86 & 15.08 \\
\hline $\mathrm{C}_{5} \mathrm{H}_{4} \mathrm{O}_{2}$ & Furfural & $98-01-1$ & 96 & 17.99 \\
\hline $\mathrm{C}_{5} \mathrm{H}_{4} \mathrm{O}_{2}$ & 3-Furaldehyde & $498-60-2$ & 96 & 18.89 \\
\hline $\mathrm{C}_{6} \mathrm{H}_{12} \mathrm{O}$ & n-Hexanal & $66-25-1$ & 100 & 19.36 \\
\hline $\mathrm{C}_{7} \mathrm{H}_{14} \mathrm{O}$ & n-Heptanal & $111-71-7$ & 114 & 23.46 \\
\hline $\mathrm{C}_{6} \mathrm{H}_{6} \mathrm{O}_{2}$ & $\begin{array}{l}\text { 5-Methyl-2- } \\
\text { furancarboxaldehyde }\end{array}$ & $620-02-0$ & 110 & 23.89 \\
\hline $\mathrm{C}_{7} \mathrm{H}_{6} \mathrm{O}$ & Benzaldehyde & $100-52-7$ & 106 & 24.61 \\
\hline $\mathrm{C}_{8} \mathrm{H}_{16} \mathrm{O}$ & 2-Ethylhexanal & $123-05-7$ & 128 & 25.86 \\
\hline $\mathrm{C}_{8} \mathrm{H}_{16} \mathrm{O}$ & n-Octanal & $124-13-0$ & 128 & 27.22 \\
\hline $\mathrm{C}_{9} \mathrm{H}_{18} \mathrm{O}$ & n-Nonanal & $124-19-6$ & 142 & 30.68 \\
\hline $\mathrm{C}_{6} \mathrm{H}_{6} \mathrm{O}_{3}$ & 5-Hydroxymethylfurfural & $67-47-0$ & 126 & 31.43 \\
\hline $\mathrm{C}_{10} \mathrm{H}_{20} \mathrm{O}$ & n-Decanal & $112-31-2$ & 156 & 33.89 \\
\hline $\mathrm{C}_{11} \mathrm{H}_{22} \mathrm{O}$ & n-Undecanal & $112-44-7$ & 170 & 37.88 \\
\hline $\mathrm{C}_{12} \mathrm{H}_{24} \mathrm{O}$ & n-Dodecanal & $112-54-9$ & 184 & 43.73 \\
\hline $\mathrm{C}_{13} \mathrm{H}_{26} \mathrm{O}$ & n-Tridecanal & $10486-19-8$ & 198 & 52.88 \\
\hline $\mathrm{C}_{14} \mathrm{H}_{28} \mathrm{O}$ & n-Tetradecanal & $124-25-4$ & 212 & 67.07 \\
\hline $\mathrm{C}_{15} \mathrm{H}_{30} \mathrm{O}$ & n-Pentadecanal & $2765-11-9$ & 226 & 89.93 \\
\hline \multicolumn{5}{|l|}{ Ketones } \\
\hline $\mathrm{C}_{3} \mathrm{H}_{6} \mathrm{O}$ & Acetone & $67-64-1$ & 58 & 7.97 \\
\hline $\mathrm{C}_{4} \mathrm{H}_{6} \mathrm{O}$ & 3-Buten-2-one & $78-94-4$ & 70 & 10.78 \\
\hline
\end{tabular}




\begin{tabular}{|c|c|c|c|c|c|}
\hline & & & & & \\
\hline $\mathrm{C}_{4} \mathrm{H}_{6} \mathrm{O}_{2}$ & 2,3-Butanedione & $431-03-8$ & 86 & 11.01 & 0.018 \\
\hline $\mathrm{C}_{4} \mathrm{H}_{8} \mathrm{O}$ & 2-Butanone & $78-93-3$ & 72 & 11.13 & 0.010 \\
\hline $\mathrm{C}_{5} \mathrm{H}_{10} \mathrm{O}$ & 2-Pentanone & $107-87-9$ & 86 & 14.85 & 0.014 \\
\hline $\mathrm{C}_{5} \mathrm{H}_{8} \mathrm{O}$ & Cyclopentanone & $120-92-3$ & 84 & 17.64 & 0.003 \\
\hline $\mathrm{C}_{6} \mathrm{H}_{12} \mathrm{O}$ & 2-Hexanone & $591-78-6$ & 100 & 19.11 & 0.011 \\
\hline $\mathrm{C}_{8} \mathrm{H}_{14} \mathrm{O}$ & 4-Octen-3-one & $14129-48-7$ & 126 & 21.05 & 0.094 \\
\hline $\mathrm{C}_{6} \mathrm{H}_{10} \mathrm{O}$ & Cyclohexanone & $108-94-1$ & 98 & 22.20 & 0.003 \\
\hline $\mathrm{C}_{7} \mathrm{H}_{14} \mathrm{O}$ & 2-Heptanone & $110-43-0$ & 114 & 23.15 & 0.026 \\
\hline $\mathrm{C}_{8} \mathrm{H}_{16} \mathrm{O}$ & 6-Methyl-2-heptanone & $928-68-7$ & 128 & 26.09 & 0.038 \\
\hline $\mathrm{C}_{8} \mathrm{H}_{14} \mathrm{O}$ & 6-Methyl-5-hepten-2-one & $110-93-0$ & 126 & 26.37 & 0.009 \\
\hline $\mathrm{C}_{8} \mathrm{H}_{16} \mathrm{O}$ & 2-Octanone & $111-13-7$ & 128 & 26.89 & 0.031 \\
\hline $\mathrm{C}_{9} \mathrm{H}_{18} \mathrm{O}$ & 2-Nonanone & $821-55-6$ & 142 & 30.35 & 0.027 \\
\hline $\mathrm{C}_{10} \mathrm{H}_{20} \mathrm{O}$ & 2-Decanone & $693-54-9$ & 156 & 33.54 & 0.041 \\
\hline $\mathrm{C}_{11} \mathrm{H}_{22} \mathrm{O}$ & 2-Undecanone & $112-12-9$ & 170 & 37.33 & 0.050 \\
\hline $\mathrm{C}_{12} \mathrm{H}_{24} \mathrm{O}$ & 2-Dodecanone & $6175-49-1$ & 184 & 42.88 & 0.034 \\
\hline $\mathrm{C}_{13} \mathrm{H}_{26} \mathrm{O}$ & 2-Tridecanone & $593-08-8$ & 198 & 51.46 & 0.046 \\
\hline $\mathrm{C}_{14} \mathrm{H}_{28} \mathrm{O}$ & 2-Tetradecanone & $2345-27-9$ & 212 & 65.06 & 0.034 \\
\hline $\mathrm{C}_{15} \mathrm{H}_{30} \mathrm{O}$ & 2-Pentadecanone & $2345-28-0$ & 226 & 86.43 & 0.103 \\
\hline Carboxyli & cids & & & & \\
\hline $\mathrm{C}_{2} \mathrm{H}_{4} \mathrm{O}_{2}$ & Acetic acid & $64-19-7$ & 60 & 11.54 & 0.178 \\
\hline $\mathrm{C}_{3} \mathrm{H}_{6} \mathrm{O}_{2}$ & n-Propanoic acid & $79-09-4$ & 74 & 16.13 & 0.014 \\
\hline $\mathrm{C}_{4} \mathrm{H}_{8} \mathrm{O}_{2}$ & n-Butanoic acid & $107-92-6$ & 88 & 19.44 & 0.057 \\
\hline $\mathrm{C}_{5} \mathrm{H}_{10} \mathrm{O}_{2}$ & 3-Methylbutanoic acid & $503-74-2$ & 102 & 22.53 & 0.006 \\
\hline $\mathrm{C}_{5} \mathrm{H}_{10} \mathrm{O}_{2}$ & n-Pentanoic acid & $109-52-4$ & 102 & 23.38 & 0.027 \\
\hline $\mathrm{C}_{6} \mathrm{H}_{12} \mathrm{O}_{2}$ & n-Hexanoic acid & $142-62-1$ & 116 & 26.75 & 0.073 \\
\hline $\mathrm{C}_{7} \mathrm{H}_{14} \mathrm{O}_{2}$ & n-Heptanoic acid & $111-14-8$ & 130 & 30.15 & 0.034 \\
\hline $\mathrm{C}_{8} \mathrm{H}_{16} \mathrm{O}_{2}$ & n-Octanoic acid & $124-07-2$ & 144 & 33.20 & 0.068 \\
\hline $\mathrm{C}_{9} \mathrm{H}_{18} \mathrm{O}_{2}$ & n-Nonanoic acid & $112-05-0$ & 158 & 36.73 & 0.081 \\
\hline $\mathrm{C}_{10} \mathrm{H}_{20} \mathrm{O}_{2}$ & n-Decanoic acid & $334-48-5$ & 172 & 42.15 & 0.067 \\
\hline $\mathrm{C}_{11} \mathrm{H}_{22} \mathrm{O}_{2}$ & n-Undecanoic acid & $112-37-8$ & 186 & 63.98 & 0.073 \\
\hline $\mathrm{C}_{11} \mathrm{H}_{14} \mathrm{O}_{3}$ & 2,4,6-Trimethylmandelic acid & $20797-56-2$ & 194 & 71.35 & 0.020 \\
\hline $\mathrm{C}_{12} \mathrm{H}_{24} \mathrm{O}_{2}$ & n-Dodecanoic acid & $143-07-7$ & 200 & 73.96 & 0.126 \\
\hline $\mathrm{C}_{13} \mathrm{H}_{26} \mathrm{O}_{2}$ & n-Tridecanoic acid & $638-53-9$ & 214 & 113.49 & 0.176 \\
\hline & Sulfona & d compounds & & & \\
\hline $\mathrm{H}_{2} \mathrm{~S}$ & Hydrogen sulfide & $7783-06-4$ & 34 & 3.46 & $<0.001$ \\
\hline COS & Carbonyl sulfide & $463-58-1$ & 60 & 3.83 & 0.006 \\
\hline $\mathrm{O}_{2} \mathrm{~S}$ & Sulfur dioxide & $7446-09-5$ & 64 & 4.96 & 0.016 \\
\hline $\mathrm{CS}_{2}$ & Carbon disulfide & $75-15-0$ & 76 & 8.33 & 0.005 \\
\hline $\mathrm{C}_{2} \mathrm{H}_{6} \mathrm{~S}$ & Dimethyl sulfide & $75-18-3$ & 62 & 8.22 & 0.004 \\
\hline $\mathrm{C}_{2} \mathrm{H}_{6} \mathrm{~S}_{2}$ & Dimethyl disulfide & $624-92-0$ & 94 & 15.85 & 0.003 \\
\hline $\mathrm{C}_{9} \mathrm{H}_{14} \mathrm{~S}$ & 2-Pentylthiophene & $4861-58-9$ & 154 & 31.29 & 0.001 \\
\hline $\mathrm{C}_{10} \mathrm{H}_{16} \mathrm{~S}$ & 2-Hexylthiophene & 18794-77-9 & 168 & 35.30 & 0.001 \\
\hline & Nitrogen & ed compound & & & \\
\hline $\mathrm{N}_{2}$ & Nitrogen & 7727-37-9 & 28 & 1.95 & 0.224 \\
\hline $\mathrm{C}_{5} \mathrm{H}_{9} \mathrm{~N}$ & 1-Isocyanobutane & $2769-64-4$ & 83 & 6.55 & 0.025 \\
\hline $\mathrm{C}_{2} \mathrm{H}_{3} \mathrm{~N}$ & Acetonitrile & $75-05-8$ & 41 & 7.17 & 0.038 \\
\hline $\mathrm{C}_{2} \mathrm{H}_{7} \mathrm{~N}$ & Dimethylamine & $124-40-3$ & 45 & 6.84 & 0.029 \\
\hline
\end{tabular}




\begin{tabular}{|c|c|c|c|c|c|}
\hline $\mathrm{CH}_{3} \mathrm{NO}$ & Formamide & $75-12-7$ & 45 & 8.37 & 0.004 \\
\hline $\mathrm{C}_{5} \mathrm{H}_{5} \mathrm{~N}$ & Pyridine & $110-86-1$ & 79 & 16.11 & 0.007 \\
\hline $\mathrm{C}_{6} \mathrm{H}_{11} \mathrm{NO}$ & 6-Methyl-2-piperidinone & $4775-98-8$ & 113 & 22.22 & 0.001 \\
\hline $\mathrm{C}_{4} \mathrm{H}_{5} \mathrm{NO}_{2}$ & Succinimide & $123-56-8$ & 99 & 29.36 & 0.074 \\
\hline \multicolumn{6}{|c|}{ Inorganic compounds } \\
\hline \multicolumn{6}{|l|}{ Oxides } \\
\hline $\mathrm{H}_{2} \mathrm{O}$ & Water & $7732-18-5$ & 18 & 3.68 & 65.673 \\
\hline $\mathrm{CO}_{2}$ & Carbon dioxide & $124-38-9$ & 44 & 2.25 & 23.486 \\
\hline
\end{tabular}

Note: ${ }^{1} \mathrm{CAS}$ - unique numerical identifier of chemical compounds included in the register Chemical Abstracts Service (https://www.cas.org); ${ }^{2} \mathrm{MW}$ - nominal mass; ${ }^{3} \mathrm{RT}$ - retention time; ${ }^{4} \mathrm{~A}$ - normalized area (the area ratio of the individual gas mixture components to the summ of the areas of all the components in the chromatogram). 
Table S3. Results of the GC-MS analysis of volatile components extracted by mechanical shock crushing from quartz from pegmatite of the Kukhilal deposit, Tajikistan.

\begin{tabular}{|c|c|c|c|c|c|}
\hline \multirow[b]{2}{*}{ Formula } & \multirow[b]{2}{*}{ Name } & \multirow[b]{2}{*}{${ }^{1} \mathrm{CAS} /(\mathrm{NIST})$} & \multirow[b]{2}{*}{${ }^{2} \mathrm{MW}$} & \multicolumn{2}{|c|}{ Quartz } \\
\hline & & & & $\begin{array}{l}{ }^{3} \mathrm{RT}, \\
\mathrm{min}\end{array}$ & ${ }^{4} \mathrm{~A}, \%$ \\
\hline \multicolumn{6}{|c|}{ Aliphatic hydrocarbons } \\
\hline \multicolumn{6}{|l|}{ Paraffins } \\
\hline $\mathrm{CH}_{4}$ & Methane & $74-82-8$ & 32 & 1.82 & 2.637 \\
\hline $\mathrm{C}_{2} \mathrm{H}_{6}$ & Ethane & $74-84-0$ & 30 & 2.45 & 0.022 \\
\hline $\mathrm{C}_{3} \mathrm{H}_{8}$ & n-Propane & $74-98-6$ & 44 & 4.28 & 0.124 \\
\hline $\mathrm{C}_{4} \mathrm{H}_{10}$ & n-Butane & $106-97-8$ & 58 & 6.16 & 0.019 \\
\hline $\mathrm{C}_{5} \mathrm{H}_{12}$ & 2-Methylbutane & $78-78-4$ & 72 & 8.18 & 0.015 \\
\hline $\mathrm{C}_{5} \mathrm{H}_{12}$ & n-Pentane & $109-66-0$ & 72 & 8.63 & 0.011 \\
\hline $\mathrm{C}_{6} \mathrm{H}_{14}$ & n-Hexane & $110-54-3$ & 86 & 12.12 & 0.025 \\
\hline $\mathrm{C}_{4} \mathrm{H}_{9} \mathrm{Cl}$ & 1-Chlorobutane & $109-69-3$ & 92 & 12.66 & 0.082 \\
\hline $\mathrm{C}_{7} \mathrm{H}_{16}$ & n-Heptane & $142-82-5$ & 100 & 16.22 & 0.023 \\
\hline $\mathrm{C}_{8} \mathrm{H}_{18}$ & n-Octane & $111-65-9$ & 114 & 20.32 & 0.152 \\
\hline $\mathrm{C}_{9} \mathrm{H}_{20}$ & n-Nonane & $111-84-2$ & 128 & 24.25 & 0.080 \\
\hline $\mathrm{C}_{10} \mathrm{H}_{22}$ & n-Decane & $124-18-5$ & 142 & 27.84 & 0.027 \\
\hline $\mathrm{C}_{11} \mathrm{H}_{24}$ & n-Undecane & $1120-21-4$ & 156 & 31.21 & 0.023 \\
\hline $\mathrm{C}_{12} \mathrm{H}_{26}$ & n-Dodecane & $112-40-3$ & 170 & 34.37 & 0.019 \\
\hline $\mathrm{C}_{13} \mathrm{H}_{28}$ & $\mathrm{n}$-Tridecane & $629-50-5$ & 184 & 38.55 & 0.039 \\
\hline $\mathrm{C}_{14} \mathrm{H}_{30}$ & n-Tetradecane & $629-59-4$ & 198 & 44.95 & 0.044 \\
\hline $\mathrm{C}_{15} \mathrm{H}_{32}$ & n-Pentadecane & $629-62-9$ & 212 & 55.00 & 0.094 \\
\hline $\mathrm{C}_{16} \mathrm{H}_{34}$ & n-Hexadecane & $544-76-3$ & 226 & 63.70 & 0.520 \\
\hline $\mathrm{C}_{17} \mathrm{H}_{36}$ & 7-Methylhexadecane & $26730-20-1$ & 240 & 71.06 & 0.183 \\
\hline $\mathrm{C}_{17} \mathrm{H}_{36}$ & n-Heptadecane & $629-78-7$ & 240 & 90.14 & 1.139 \\
\hline $\mathrm{C}_{18} \mathrm{H}_{38}$ & 7-Methylheptadecane & $20959-33-5$ & 254 & 97.32 & 0.242 \\
\hline $\mathrm{C}_{18} \mathrm{H}_{38}$ & 4-Methylheptadecane & $26429-11-8$ & 254 & 121.68 & 0.392 \\
\hline $\mathrm{C}_{18} \mathrm{H}_{38}$ & 2-Methylheptadecane & $1560-89-0$ & 254 & 128.48 & 0.158 \\
\hline $\mathrm{C}_{18} \mathrm{H}_{38}$ & 3-Methylheptadecane & $6418-44-6$ & 254 & 132.89 & 0.234 \\
\hline \multicolumn{6}{|l|}{ Olefins } \\
\hline $\mathrm{C}_{2} \mathrm{H}_{2}$ & Acetylene & $74-86-2$ & 26 & 2.28 & 0.009 \\
\hline $\mathrm{C}_{3} \mathrm{H}_{6}$ & 1-Propene & $115-07-1$ & 42 & 4.06 & 0.038 \\
\hline $\mathrm{C}_{4} \mathrm{H}_{8}$ & 1-Butene & $106-98-9$ & 56 & 5.83 & 0.106 \\
\hline $\mathrm{C}_{4} \mathrm{H}_{8}$ & 2-Methyl-1-propene & $115-11-7$ & 56 & 5.95 & 0.191 \\
\hline $\mathrm{C}_{4} \mathrm{H}_{8}$ & (E)-2-Butene & $624-64-6$ & 56 & 6.16 & 0.051 \\
\hline $\mathrm{C}_{4} \mathrm{H}_{8}$ & 2-Butene & $107-01-7$ & 56 & 6.26 & 0.055 \\
\hline $\mathrm{C}_{5} \mathrm{H}_{8}$ & 1,4-Pentadiene & $591-93-5$ & 68 & 7.98 & 0.001 \\
\hline $\mathrm{C}_{5} \mathrm{H}_{10}$ & 1-Pentene & $109-67-1$ & 70 & 8.33 & 0.023 \\
\hline $\mathrm{C}_{5} \mathrm{H}_{8}$ & 1,3-Pentadiene & $504-60-9$ & 68 & 8.54 & 0.011 \\
\hline $\mathrm{C}_{5} \mathrm{H}_{10}$ & 2-Methyl-2-butene & $513-35-9$ & 70 & 8.69 & 0.022 \\
\hline $\mathrm{C}_{5} \mathrm{H}_{8}$ & (Z)-1,3-Pentadiene & $1574-41-0$ & 68 & 8.76 & 0.002 \\
\hline $\mathrm{C}_{5} \mathrm{H}_{8}$ & 1,2-Pentadiene & $591-95-7$ & 68 & 9.06 & 0.002 \\
\hline $\mathrm{C}_{5} \mathrm{H}_{8}$ & 2,3-Pentadiene & $591-96-8$ & 68 & 9.13 & 0.001 \\
\hline $\mathrm{C}_{6} \mathrm{H}_{12}$ & 1-Hexene & $592-41-6$ & 84 & 11.77 & 0.025 \\
\hline $\mathrm{C}_{6} \mathrm{H}_{10}$ & 4-Methyl-1,3-pentadiene & $926-56-7$ & 82 & 13.11 & 0.003 \\
\hline $\mathrm{C}_{6} \mathrm{H}_{10}$ & (Z)-3-Methyl-1,3-pentadiene & $2787-45-3$ & 82 & 13.46 & 0.002 \\
\hline $\mathrm{C}_{7} \mathrm{H}_{12}$ & 5-Methyl-1-hexyne & $2203-80-7$ & 96 & 14.11 & 0.001 \\
\hline
\end{tabular}




\begin{tabular}{l|l}
$\mathrm{C}_{7} \mathrm{H}_{12}$ & 1-Heptyne \\
$\mathrm{C}_{7} \mathrm{H}_{14}$ & 1-Heptene \\
$\mathrm{C}_{8} \mathrm{H}_{16}$ & 2,3-Dimethyl-3-hexene \\
$\mathrm{C}_{8} \mathrm{H}_{16}$ & 5-Methyl-3-heptene \\
$\mathrm{C}_{8} \mathrm{H}_{16}$ & 2,5-Dimethyl-2-hexene \\
$\mathrm{C}_{8} \mathrm{H}_{16}$ & (E)-4-Octene \\
$\mathrm{C}_{8} \mathrm{H}_{16}$ & (Z)-3-Octene \\
$\mathrm{C}_{8} \mathrm{H}_{16}$ & 2,3-Dimethyl-1-hexene \\
$\mathrm{C}_{8} \mathrm{H}_{16}$ & (E)-2-Octene \\
$\mathrm{C}_{9} \mathrm{H}_{18}$ & 1-Nonene \\
$\mathrm{C}_{10} \mathrm{H}_{20}$ & 1-Decene \\
$\mathrm{C}_{11} \mathrm{H}_{22}$ & 1-Undecene \\
$\mathrm{C}_{12} \mathrm{H}_{24}$ & 1-Dodecene \\
$\mathrm{C}_{13} \mathrm{H}_{26}$ & 1-Tridecene \\
$\mathrm{C}_{14} \mathrm{H}_{28}$ & 1-Tetradecene \\
$\mathrm{C}_{15} \mathrm{H}_{30}$ & 1-Pentadecene \\
$\mathrm{C}_{16} \mathrm{H}_{32}$ & 1-Hexadecene
\end{tabular}

\begin{tabular}{|l|c|c|c|}
$628-71-7$ & 96 & 15.04 & 0.003 \\
$592-76-7$ & 98 & 15.84 & 0.023 \\
$7145-23-5$ & 112 & 19.53 & 0.120 \\
$13172-91-3$ & 114 & 19.68 & 0.313 \\
$3404-78-2$ & 112 & 19.88 & 0.200 \\
$14850-23-8$ & 112 & 19.97 & 0.067 \\
$14850-22-7$ & 112 & 20.02 & 0.245 \\
$16746-86-4$ & 112 & 20.18 & 0.104 \\
$13389-42-9$ & 112 & 20.43 & 0.026 \\
$124-11-8$ & 126 & 23.96 & 0.058 \\
$872-05-9$ & 140 & 27.61 & 0.006 \\
$821-95-4$ & 154 & 31.08 & 0.024 \\
$112-41-4$ & 168 & 34.14 & 0.014 \\
$2437-56-1$ & 182 & 38.29 & 0.030 \\
$1120-36-1$ & 196 & 44.55 & 0.031 \\
$13360-61-7$ & 210 & 54.32 & 0.034 \\
$629-73-2$ & 224 & 79.47 & 0.055
\end{tabular}

Cyclic alkanes and alkenes

\begin{tabular}{l|l}
$\mathrm{C}_{6} \mathrm{H}_{10}$ & 4-Methylcyclopentene \\
$\mathrm{C}_{7} \mathrm{H}_{12}$ & 1-Methyl-2- \\
methylenecyclopentane \\
$\mathrm{C}_{7} \mathrm{H}_{14}$ & Methylcyclohexane \\
$\mathrm{C}_{8} \mathrm{H}_{14}$ & 3-Propylcyclopentene \\
Arenes & \\
$\mathrm{C}_{6} \mathrm{H}_{6}$ & Benzene \\
$\mathrm{C}_{7} \mathrm{H}_{8}$ & Toluene \\
$\mathrm{C}_{8} \mathrm{H}_{10}$ & Ethylbenzene \\
$\mathrm{C}_{8} \mathrm{H}_{10}$ & p-Xylene \\
$\mathrm{C}_{8} \mathrm{H}_{8}$ & Styrene \\
$\mathrm{C}_{9} \mathrm{H}_{12}$ & Propylbenzene \\
$\mathrm{C}_{10} \mathrm{H}_{14}$ & o-Cymene \\
$\mathrm{C}_{10} \mathrm{H}_{14}$ & Butylbenzene \\
$\mathrm{C}_{11} \mathrm{H}_{16}$ & Pentylbenzene \\
$\mathrm{C}_{10} \mathrm{H}_{12}$ & 2-Butenylbenzene \\
$\mathrm{C}_{12} \mathrm{H}_{18}$ & Hexylbenzene \\
$\mathrm{C}_{13} \mathrm{H}_{20}$ & Heptylbenzene \\
$\mathrm{C}_{14} \mathrm{H}_{22}$ & Octylbenzene \\
$\mathrm{C}_{15} \mathrm{H}_{24}$ & Nonylbenzene
\end{tabular}

\section{Cyclic hydrocarbons}

\begin{tabular}{|l|c|c|c}
$1759-81-5$ & 82 & 12.22 & 0.014 \\
$41158-41-2$ & 96 & 16.10 & 0.007 \\
$108-87-2$ & 98 & 18.12 & 0.003 \\
$34067-75-9$ & 110 & 21.25 & 0.009
\end{tabular}

Polycyclic aromatic hydrocarbons

\begin{tabular}{|c|c|c|c|}
\hline $71-43-2$ & 78 & 12.57 & 0.023 \\
\hline $108-88-3$ & 92 & 17.07 & 0.083 \\
\hline $100-41-4$ & 106 & 21.15 & 0.002 \\
\hline $106-42-3$ & 106 & 21.42 & 0.006 \\
\hline $100-42-5$ & 104 & 22.03 & 0.006 \\
\hline $103-65-1$ & 120 & 25.00 & 0.005 \\
\hline $527-84-4$ & 134 & 27.88 & 0.044 \\
\hline $104-51-8$ & 134 & 28.79 & 0.007 \\
\hline 538-68-1 & 148 & 32.22 & 0.010 \\
\hline $1560-06-1$ & 132 & 34.07 & 0.009 \\
\hline $1077-16-3$ & 162 & 35.72 & 0.008 \\
\hline $1078-71-3$ & 176 & 38.83 & 0.044 \\
\hline $2189-60-8$ & 190 & 45.51 & 0.017 \\
\hline $1081-77-2$ & 204 & 61.08 & 0.028 \\
\hline $91-20-3$ & 128 & 32.27 & 0.003 \\
\hline $91-57-6$ & 142 & 36.14 & 0.003 \\
\hline $90-12-0$ & 142 & 36.64 & 0.002 \\
\hline
\end{tabular}

\section{Heterocyclic hydrocarbons}

Dioxanes

\begin{tabular}{l|l}
$\mathrm{C}_{4} \mathrm{H}_{8} \mathrm{O}_{2}$ & 1,4-Dioxane \\
$\mathrm{C}_{4} \mathrm{H}_{8} \mathrm{O}_{2}$ & 1,3-Dioxane
\end{tabular}

$\mid \begin{aligned} & 123-91-1 \\ & 505-22-6\end{aligned}$

88
88

13.64

0.001

Furans 


\begin{tabular}{l|l}
$\mathrm{C}_{5} \mathrm{H}_{6} \mathrm{O}$ & 2-Methylfuran \\
$\mathrm{C}_{5} \mathrm{H}_{6} \mathrm{O}$ & 3-Methylfuran \\
$\mathrm{C}_{6} \mathrm{H}_{8} \mathrm{O}$ & 2-Ethylfuran \\
$\mathrm{C}_{7} \mathrm{H}_{10} \mathrm{O}$ & 2-Propylfuran \\
$\mathrm{C}_{8} \mathrm{H}_{12} \mathrm{O}$ & 2-Butylfuran \\
$\mathrm{C}_{9} \mathrm{H}_{14} \mathrm{O}$ & 2-Pentylfuran \\
$\mathrm{C}_{10} \mathrm{H}_{16} \mathrm{O}$ & 2-Hexylfuran \\
$\mathrm{C}_{11} \mathrm{H}_{18} \mathrm{O}$ & 2-Heptylfuran \\
$\mathrm{C}_{12} \mathrm{H}_{20} \mathrm{O}$ & 2-Octylfuran \\
$\mathrm{C}_{15} \mathrm{H}_{26} \mathrm{O}$ & 2-Decylfuran
\end{tabular}

Alcohols, ethers and esters

\begin{tabular}{l|l}
$\mathrm{CH}_{4} \mathrm{O}$ & Methanol \\
$\mathrm{C}_{2} \mathrm{H}_{6} \mathrm{O}$ & Ethanol \\
$\mathrm{C}_{4} \mathrm{H}_{10} \mathrm{O}$ & Isobutanol \\
$\mathrm{C}_{4} \mathrm{H}_{10} \mathrm{O}$ & 1-Butanol \\
$\mathrm{C}_{5} \mathrm{H}_{8} \mathrm{O}_{2}$ & Methyl methacrylate \\
$\mathrm{C}_{4} \mathrm{H}_{6} \mathrm{O}_{2}$ & $\gamma$-Butyrolactone \\
$\mathrm{C}_{6} \mathrm{H}_{10} \mathrm{O}$ & 2-Cyclohexen-1-ol \\
$\mathrm{C}_{6} \mathrm{H}_{6} \mathrm{O}$ & Phenol \\
$\mathrm{C}_{6} \mathrm{H}_{10} \mathrm{O}_{2}$ & $\gamma$-Caprolactone \\
$\mathrm{C}_{8} \mathrm{H}_{18} \mathrm{O}$ & 2-Ethyl-1-hexanol \\
$\mathrm{C}_{7} \mathrm{H}_{12} \mathrm{O}_{2}$ & $\gamma$-Heptanolactone \\
$\mathrm{C}_{8} \mathrm{H}_{10} \mathrm{O}_{2}$ & 2-Phenoxyethanol \\
$\mathrm{C}_{8} \mathrm{H}_{14} \mathrm{O}_{2}$ & $\gamma$-Octalactone \\
$\mathrm{C}_{10} \mathrm{H}_{20} \mathrm{O}$ & (E)-2-Decen-1-ol \\
$\mathrm{C}_{9} \mathrm{H}_{16} \mathrm{O}_{2}$ & $\gamma$-Nonalactone \\
$\mathrm{C}_{10} \mathrm{H}_{18} \mathrm{O}_{2}$ & $\gamma$-Decalactone \\
$\mathrm{C}_{11} \mathrm{H}_{20} \mathrm{O}_{2}$ & $\gamma$-Undecalactone \\
$\mathrm{C}_{12} \mathrm{H}_{14} \mathrm{O}_{4}$ & Diethyl Phthalate \\
$\mathrm{C}_{14} \mathrm{H}_{18} \mathrm{O}_{4}$ & Diisopropyl phthalate \\
$\mathrm{C}_{12} \mathrm{H}_{22} \mathrm{O}_{2}$ & $\gamma$-Dodecalactone \\
$\mathrm{C}_{13} \mathrm{H}_{24} \mathrm{O}_{2}$ & $\delta$-Tridecalactone \\
${ }_{\text {Aldehydes }}$ & \\
$\mathrm{C}_{2} \mathrm{H}_{4} \mathrm{O}$ & Acetaldehyde \\
$\mathrm{C}_{3} \mathrm{H}_{4} \mathrm{O}$ & 2-Propenal \\
$\mathrm{C}_{3} \mathrm{H}_{6} \mathrm{O}$ & n-Propanal \\
$\mathrm{C}_{4} \mathrm{H}_{6} \mathrm{O}$ & 2-Methyl-2-propenal \\
$\mathrm{C}_{4} \mathrm{H}_{8} \mathrm{O}$ & 2-Methylpropanal \\
$\mathrm{C}_{4} \mathrm{H}_{8} \mathrm{O}$ & n-Butanal \\
$\mathrm{C}_{5} \mathrm{H}_{10} \mathrm{O}$ & 3-Methylbutanal \\
$\mathrm{C}_{5} \mathrm{H}_{10} \mathrm{O}$ & n-Pentanal \\
$\mathrm{C}_{5} \mathrm{H}_{4} \mathrm{O}_{2}$ & Furfural \\
$\mathrm{C}_{5} \mathrm{H}_{4} \mathrm{O}_{2}$ & 3-Furaldehyde \\
$\mathrm{C}_{6} \mathrm{H}_{12} \mathrm{O}$ & n-Hexanal \\
$\mathrm{C}_{7} \mathrm{H}_{14} \mathrm{O}$ & n-Heptanal \\
$\mathrm{C}_{6} \mathrm{H}_{6} \mathrm{O}_{2}$ & 5-Methyl-2- \\
& furancarboxaldehyde
\end{tabular}

\begin{tabular}{|l|c|c|c|}
$534-22-5$ & 82 & 10.31 & 0.006 \\
$930-27-8$ & 92 & 10.56 & 0.001 \\
$3208-16-0$ & 96 & 12.22 & 0.002 \\
$4229-91-8$ & 110 & 18.59 & 0.002 \\
$4466-24-4$ & 124 & 22.37 & 0.005 \\
$3777-69-3$ & 138 & 26.25 & 0.014 \\
$3777-70-6$ & 152 & 29.84 & 0.002 \\
$3777-71-7$ & 166 & 32.94 & 0.001 \\
$4179-38-8$ & 180 & 36.84 & 0.008 \\
$83469-85-6$ & 222 & 69.24 & 0.010
\end{tabular}

Oxygenated hydrocarbons

\begin{tabular}{|l|c|c|c|}
$67-56-1$ & 32 & 4.88 & 0.054 \\
$64-17-5$ & 46 & 6.45 & 0.034 \\
$78-83-1$ & 74 & 11.96 & 0.002 \\
$71-36-3$ & 74 & 12.82 & 0.162 \\
$80-62-6$ & 100 & 14.49 & 0.026 \\
$96-48-0$ & 86 & 20.78 & 0.120 \\
$822-67-3$ & 98 & 21.65 & 0.002 \\
$108-95-2$ & 94 & 24.71 & 0.020 \\
$695-06-7$ & 114 & 27.41 & 0.006 \\
$104-76-7$ & 130 & 27.99 & 0.199 \\
$105-21-5$ & 128 & 32.11 & 0.005 \\
$122-99-6$ & 138 & 33.09 & 0.067 \\
$104-50-7$ & 142 & 34.57 & 0.010 \\
$18409-18-2$ & 156 & 36.97 & 0.041 \\
$104-61-0$ & 156 & 39.13 & 0.017 \\
$706-14-9$ & 170 & 46.16 & 0.012 \\
$104-67-6$ & 184 & 57.22 & 0.007 \\
$84-66-2$ & 222 & 58.93 & 0.026 \\
$605-45-8$ & 250 & 70.11 & 0.084 \\
$2305-05-7$ & 198 & 75.00 & 0.022 \\
$7370-92-5$ & 212 & 108.33 & 0.075
\end{tabular}

\begin{tabular}{|l|c|c|c}
$75-07-0$ & 44 & 5.21 & 0.086 \\
$107-02-8$ & 56 & 7.19 & 0.007 \\
$123-38-6$ & 58 & 7.39 & 0.072 \\
$78-85-3$ & 70 & 9.66 & 0.023 \\
$78-84-2$ & 72 & 9.73 & 0.012 \\
$123-72-8$ & 72 & 10.49 & 0.003 \\
$590-86-3$ & 86 & 13.69 & 0.134 \\
$110-62-3$ & 86 & 14.69 & 0.022 \\
$98-01-1$ & 96 & 17.35 & 0.003 \\
$498-60-2$ & 96 & 18.24 & 0.024 \\
$66-25-1$ & 100 & 19.04 & 0.206 \\
$111-71-7$ & 114 & 23.18 & 0.146 \\
$620-02-0$ & 110 & 23.30 & 0.006
\end{tabular}




\begin{tabular}{|c|c|c|c|c|c|}
\hline $\mathrm{C}_{7} \mathrm{H}_{6} \mathrm{O}$ & Benzaldehyde & $100-52-7$ & 106 & 24.05 & 0.032 \\
\hline $\mathrm{C}_{8} \mathrm{H}_{16} \mathrm{O}$ & 2-Ethylhexanal & $123-05-7$ & 128 & 25.78 & 0.034 \\
\hline $\mathrm{C}_{8} \mathrm{H}_{16} \mathrm{O}$ & n-Octanal & $124-13-0$ & 128 & 27.06 & 0.035 \\
\hline $\mathrm{C}_{9} \mathrm{H}_{18} \mathrm{O}$ & n-Nonanal & $124-19-6$ & 142 & 30.58 & 0.055 \\
\hline $\mathrm{C}_{6} \mathrm{H}_{6} \mathrm{O}_{3}$ & 5-Hydroxymethylfurfural & $67-47-0$ & 126 & 31.96 & 0.023 \\
\hline $\mathrm{C}_{10} \mathrm{H}_{20} \mathrm{O}$ & n-Decanal & $112-31-2$ & 156 & 33.87 & 0.042 \\
\hline $\mathrm{C}_{11} \mathrm{H}_{22} \mathrm{O}$ & n-Undecanal & $112-44-7$ & 170 & 37.92 & 0.055 \\
\hline $\mathrm{C}_{12} \mathrm{H}_{24} \mathrm{O}$ & n-Dodecanal & $112-54-9$ & 184 & 44.03 & 0.018 \\
\hline $\mathrm{C}_{13} \mathrm{H}_{26} \mathrm{O}$ & n-Tridecanal & $10486-19-8$ & 198 & 53.84 & 0.024 \\
\hline $\mathrm{C}_{14} \mathrm{H}_{28} \mathrm{O}$ & n-Tetradecanal & $124-25-4$ & 212 & 61.85 & 0.072 \\
\hline \multicolumn{6}{|l|}{ Ketones } \\
\hline $\mathrm{C}_{3} \mathrm{H}_{6} \mathrm{O}$ & Acetone & $67-64-1$ & 58 & 7.56 & 0.137 \\
\hline $\mathrm{C}_{4} \mathrm{H}_{6} \mathrm{O}_{2}$ & 2,3-Butanedione & $431-03-8$ & 86 & 10.58 & 0.003 \\
\hline $\mathrm{C}_{4} \mathrm{H}_{8} \mathrm{O}$ & 2-Butanone & $78-93-3$ & 72 & 10.64 & 0.004 \\
\hline $\mathrm{C}_{5} \mathrm{H}_{10} \mathrm{O}$ & 2-Pentanone & $107-87-9$ & 86 & 14.46 & 0.013 \\
\hline $\mathrm{C}_{5} \mathrm{H}_{8} \mathrm{O}$ & Cyclopentanone & $120-92-3$ & 84 & 16.82 & 0.002 \\
\hline $\mathrm{C}_{6} \mathrm{H}_{12} \mathrm{O}$ & 2-Hexanone & $591-78-6$ & 100 & 18.79 & 0.007 \\
\hline $\mathrm{C}_{7} \mathrm{H}_{14} \mathrm{O}$ & 2-Heptanone & $110-43-0$ & 114 & 22.92 & 0.025 \\
\hline $\mathrm{C}_{8} \mathrm{H}_{16} \mathrm{O}$ & 6-Methyl-2-heptanone & $928-68-7$ & 128 & 25.95 & 0.011 \\
\hline $\mathrm{C}_{8} \mathrm{H}_{14} \mathrm{O}$ & 6-Methyl-5-hepten-2-one & $110-93-0$ & 126 & 26.20 & 0.017 \\
\hline $\mathrm{C}_{8} \mathrm{H}_{16} \mathrm{O}$ & 2-Octanone & $111-13-7$ & 128 & 26.73 & 0.030 \\
\hline $\mathrm{C}_{9} \mathrm{H}_{18} \mathrm{O}$ & 2-Nonanone & $821-55-6$ & 142 & 30.28 & 0.024 \\
\hline $\mathrm{C}_{10} \mathrm{H}_{20} \mathrm{O}$ & 2-Decanone & $693-54-9$ & 156 & 33.52 & 0.029 \\
\hline $\mathrm{C}_{8} \mathrm{H}_{4} \mathrm{O}_{3}$ & Phthalic anhydride & $85-44-9$ & 148 & 35.82 & 0.101 \\
\hline $\mathrm{C}_{11} \mathrm{H}_{22} \mathrm{O}$ & 2-Undecanone & $112-12-9$ & 170 & 37.39 & 0.048 \\
\hline $\mathrm{C}_{12} \mathrm{H}_{24} \mathrm{O}$ & 2-Dodecanone & $6175-49-1$ & 184 & 43.16 & 0.024 \\
\hline $\mathrm{C}_{13} \mathrm{H}_{26} \mathrm{O}$ & 2-Tridecanone & $593-08-8$ & 198 & 52.32 & 0.027 \\
\hline $\mathrm{C}_{14} \mathrm{H}_{28} \mathrm{O}$ & 2-Tetradecanone & $2345-27-9$ & 212 & 59.55 & 0.040 \\
\hline $\mathrm{C}_{15} \mathrm{H}_{30} \mathrm{O}$ & 2-Pentadecanone & $2345-28-0$ & 226 & 83.46 & 0.157 \\
\hline \multicolumn{6}{|c|}{ Carboxylic acids } \\
\hline $\mathrm{C}_{2} \mathrm{H}_{4} \mathrm{O}_{2}$ & Acetic acid & $64-19-7$ & 60 & 11.26 & 0.383 \\
\hline $\mathrm{C}_{3} \mathrm{H}_{6} \mathrm{O}_{2}$ & n-Propanoic acid & $79-09-4$ & 74 & 15.22 & 0.016 \\
\hline $\mathrm{C}_{4} \mathrm{H}_{8} \mathrm{O}_{2}$ & n-Butanoic acid & $107-92-6$ & 88 & 19.04 & 0.073 \\
\hline $\mathrm{C}_{5} \mathrm{H}_{10} \mathrm{O}_{2}$ & 3-Methylbutanoic acid & $503-74-2$ & 102 & 22.13 & 0.012 \\
\hline $\mathrm{C}_{5} \mathrm{H}_{10} \mathrm{O}_{2}$ & n-Pentanoic acid & $109-52-4$ & 102 & 23.03 & 0.056 \\
\hline $\mathrm{C}_{6} \mathrm{H}_{12} \mathrm{O}_{2}$ & n-Hexanoic acid & $142-62-1$ & 116 & 26.51 & 0.292 \\
\hline $\mathrm{C}_{7} \mathrm{H}_{14} \mathrm{O}_{2}$ & n-Heptanoic acid & $111-14-8$ & 130 & 30.01 & 0.124 \\
\hline $\mathrm{C}_{8} \mathrm{H}_{16} \mathrm{O}_{2}$ & n-Octanoic acid & $124-07-2$ & 144 & 33.12 & 0.189 \\
\hline $\mathrm{C}_{9} \mathrm{H}_{18} \mathrm{O}_{2}$ & n-Nonanoic acid & $112-05-0$ & 158 & 36.69 & 0.153 \\
\hline $\mathrm{C}_{10} \mathrm{H}_{20} \mathrm{O}_{2}$ & n-Decanoic acid & $334-48-5$ & 172 & 42.22 & 0.152 \\
\hline $\mathrm{C}_{11} \mathrm{H}_{22} \mathrm{O}_{2}$ & n-Undecanoic acid & $112-37-8$ & 186 & 51.79 & 0.034 \\
\hline $\mathrm{C}_{12} \mathrm{H}_{24} \mathrm{O}_{2}$ & n-Dodecanoic acid & $143-07-7$ & 200 & 65.23 & 0.118 \\
\hline $\mathrm{C}_{13} \mathrm{H}_{26} \mathrm{O}_{2}$ & n-Tetradecanoic acid & $544-63-8$ & 214 & 123.18 & 0.127 \\
\hline \multicolumn{6}{|c|}{ Sulfonated compounds } \\
\hline $\mathrm{H}_{2} \mathrm{~S}$ & Hydrogen sulfide & $7783-06-4$ & 34 & 2.83 & 0.128 \\
\hline $\mathrm{COS}$ & Carbonyl sulfide & $463-58-1$ & 60 & 3.53 & 0.045 \\
\hline $\mathrm{O}_{2} \mathrm{~S}$ & Sulfur dioxide & $7446-09-5$ & 64 & 4.73 & 0.059 \\
\hline
\end{tabular}




\begin{tabular}{|c|c|c|c|c|c|}
\hline $\mathrm{CH}_{4} \mathrm{~S}$ & Methanethiol & $74-93-1$ & 48 & 5.50 & 0.249 \\
\hline $\mathrm{CS}_{2}$ & Carbon disulfide & $75-15-0$ & 76 & 7.83 & 0.039 \\
\hline $\mathrm{C}_{2} \mathrm{H}_{6} \mathrm{~S}$ & Dimethyl sulfide & $75-18-3$ & 62 & 7.78 & 1.817 \\
\hline $\mathrm{C}_{2} \mathrm{H}_{6} \mathrm{~S}_{2}$ & Dimethyl disulfide & $624-92-0$ & 94 & 15.37 & 0.048 \\
\hline $\mathrm{C}_{10} \mathrm{H}_{16} \mathrm{~S}$ & 2-Hexylthiophene & $18794-77-9$ & 168 & 35.05 & 0.001 \\
\hline \multicolumn{6}{|c|}{ Nitrogenated compounds } \\
\hline $\mathrm{N}_{2}$ & Nitrogen & 7727-37-9 & 28 & 1.62 & 0.099 \\
\hline $\mathrm{H}_{3} \mathrm{~N}$ & Ammonia & $7664-41-7$ & 17 & 2.88 & 0.059 \\
\hline $\mathrm{C}_{2} \mathrm{H}_{3} \mathrm{~N}$ & 2H-Azirine & $157-16-4$ & 41 & 6.80 & 0.016 \\
\hline $\mathrm{C}_{5} \mathrm{H}_{5} \mathrm{~N}$ & Pyridine & $110-86-1$ & 79 & 15.37 & 0.028 \\
\hline $\mathrm{C}_{6} \mathrm{H}_{9} \mathrm{~N}$ & 2,3-Dimethyl-1H-pyrrole & $600-28-2$ & 95 & 18.65 & 0.002 \\
\hline $\mathrm{C}_{6} \mathrm{H}_{11} \mathrm{NO}$ & 6-Methyl-2-piperidinone & $4775-98-8$ & 113 & 21.93 & 0.001 \\
\hline $\mathrm{C}_{4} \mathrm{H}_{5} \mathrm{NO}_{2}$ & Succinimide & $123-56-8$ & 99 & 28.68 & 0.009 \\
\hline $\mathrm{C}_{5} \mathrm{H}_{9} \mathrm{NO}$ & 1-Methyl-2-pyrrolidinone & $872-50-4$ & 99 & 29.89 & 0.006 \\
\hline $\mathrm{C}_{9} \mathrm{H}_{9} \mathrm{NS}$ & $\begin{array}{l}\text { 3-Methyl-2-methylene- } \\
\text { benzothiazoline } \\
\text { (2-Methyl-3- }\end{array}$ & $23574-67-6$ & 163 & 51.09 & 0.038 \\
\hline $\mathrm{C}_{10} \mathrm{H}_{11} \mathrm{NO}_{4}$ & $\begin{array}{l}\text { nitrophenyl)methyl ester acetic } \\
\text { acid }\end{array}$ & $(367949)$ & 209 & 86.41 & 0.082 \\
\hline \multicolumn{6}{|c|}{ Inorganic compounds } \\
\hline \multicolumn{6}{|l|}{ Oxides } \\
\hline $\mathrm{H}_{2} \mathrm{O}$ & Water & $7732-18-5$ & 18 & 3.20 & 76.171 \\
\hline $\mathrm{CO}_{2}$ & Carbon dioxide & $124-38-9$ & 44 & 1.90 & 7.958 \\
\hline
\end{tabular}

Note: ${ }^{1} \mathrm{CAS} /(\mathrm{NIST})$ - unique numerical identifier of chemical compounds included in the register Chemical Abstracts Service (https://www.cas.org) or NIST number (a unique number given to each spectrum in the NIST archive); ${ }^{2} \mathrm{MW}$ - nominal mass; ${ }^{3} \mathrm{RT}$ - retention time; ${ }^{4} \mathrm{~A}-$ normalized area (the area ratio of the individual gas mixture components to the summ of the areas of all the components in the chromatogram). 
Table S4. Results of the GC-MS analysis of volatile components extracted by mechanical shock crushing from tourmaline from pegmatite of the Kukhilal field, Tajikistan.

\begin{tabular}{|c|c|c|c|c|c|}
\hline \multirow{2}{*}{ Formula } & \multirow{2}{*}{ Name } & \multirow{2}{*}{${ }^{1} \mathrm{CAS}$} & \multirow{2}{*}{${ }^{2} \mathrm{MW}$} & \multicolumn{2}{|c|}{ Tourmaline } \\
\hline & & & & ${ }^{3} \mathrm{RT}, \min$ & ${ }^{4} \mathrm{~A}, \%$ \\
\hline \multicolumn{6}{|c|}{ Aliphatic hydrocarbons } \\
\hline \multicolumn{6}{|l|}{ Paraffins } \\
\hline $\mathrm{CH}_{4}$ & Methane & $74-82-8$ & 32 & 1.73 & 0.011 \\
\hline $\mathrm{C}_{2} \mathrm{H}_{6}$ & Ethane & $74-84-0$ & 30 & 2.42 & 0.018 \\
\hline $\mathrm{C}_{3} \mathrm{H}_{8}$ & n-Propane & $74-98-6$ & 44 & 4.18 & 0.012 \\
\hline $\mathrm{C}_{4} \mathrm{H}_{10}$ & n-Butane & $106-97-8$ & 58 & 6.08 & 0.056 \\
\hline $\mathrm{C}_{5} \mathrm{H}_{12}$ & n-Pentane & $109-66-0$ & 72 & 8.48 & 0.017 \\
\hline $\mathrm{C}_{6} \mathrm{H}_{14}$ & n-Hexane & $110-54-3$ & 86 & 11.86 & 0.035 \\
\hline $\mathrm{C}_{7} \mathrm{H}_{16}$ & n-Heptane & $142-82-5$ & 100 & 15.87 & 0.037 \\
\hline $\mathrm{C}_{8} \mathrm{H}_{16}$ & 2-Methylheptane & $592-27-8$ & 114 & 19.18 & 0.015 \\
\hline $\mathrm{C}_{8} \mathrm{H}_{18}$ & n-Octane & $111-65-9$ & 114 & 19.95 & 0.072 \\
\hline $\mathrm{C}_{9} \mathrm{H}_{20}$ & n-Nonane & $111-84-2$ & 128 & 23.81 & 0.126 \\
\hline $\mathrm{C}_{10} \mathrm{H}_{22}$ & n-Decane & $124-18-5$ & 142 & 27.39 & 0.062 \\
\hline $\mathrm{C}_{11} \mathrm{H}_{24}$ & n-Undecane & $1120-21-4$ & 156 & 30.71 & 0.090 \\
\hline $\mathrm{C}_{12} \mathrm{H}_{26}$ & n-Dodecane & $112-40-3$ & 170 & 33.81 & 0.088 \\
\hline $\mathrm{C}_{13} \mathrm{H}_{28}$ & $\mathrm{n}$-Tridecane & $629-50-5$ & 184 & 37.65 & 0.052 \\
\hline $\mathrm{C}_{14} \mathrm{H}_{30}$ & n-Tetradecane & $629-59-4$ & 198 & 43.43 & 0.049 \\
\hline $\mathrm{C}_{15} \mathrm{H}_{32}$ & n-Pentadecane & $629-62-9$ & 212 & 52.47 & 0.059 \\
\hline $\mathrm{C}_{16} \mathrm{H}_{34}$ & n-Hexadecane & $544-76-3$ & 226 & 66.76 & 0.080 \\
\hline $\mathrm{C}_{17} \mathrm{H}_{36}$ & n-Heptadecane & $629-78-7$ & 240 & 89.98 & 0.073 \\
\hline $\mathrm{C}_{18} \mathrm{H}_{38}$ & 7-Methylheptadecane & $20959-33-5$ & 254 & 96.64 & 0.085 \\
\hline $\mathrm{C}_{18} \mathrm{H}_{38}$ & 4-Methylheptadecane & $26429-11-8$ & 254 & 116.99 & 0.068 \\
\hline $\mathrm{C}_{18} \mathrm{H}_{38}$ & 2-Methylheptadecane & $1560-89-0$ & 254 & 126.91 & 0.075 \\
\hline \multicolumn{6}{|l|}{ Olefins } \\
\hline $\mathrm{C}_{2} \mathrm{H}_{2}$ & Acetylene & $74-86-2$ & 26 & 2.23 & 0.014 \\
\hline $\mathrm{C}_{3} \mathrm{H}_{6}$ & 1-Propene & $115-07-1$ & 42 & 4.03 & 0.049 \\
\hline $\mathrm{C}_{4} \mathrm{H}_{8}$ & 1-Butene & $106-98-9$ & 56 & 5.90 & 0.201 \\
\hline $\mathrm{C}_{4} \mathrm{H}_{8}$ & 2-Methyl-1-propene & $115-11-7$ & 56 & 6.11 & 0.021 \\
\hline $\mathrm{C}_{4} \mathrm{H}_{8}$ & (E)-2-Butene & $624-64-6$ & 56 & 6.21 & 0.015 \\
\hline $\mathrm{C}_{5} \mathrm{H}_{8}$ & 1,4-Pentadiene & $591-93-5$ & 68 & 7.88 & 0.001 \\
\hline $\mathrm{C}_{5} \mathrm{H}_{10}$ & 1-Pentene & $109-67-1$ & 70 & 8.19 & 0.044 \\
\hline $\mathrm{C}_{5} \mathrm{H}_{8}$ & 1,3-Pentadiene & $504-60-9$ & 68 & 8.44 & 0.013 \\
\hline $\mathrm{C}_{5} \mathrm{H}_{10}$ & 2-Methyl-2-butene & $513-35-9$ & 70 & 8.56 & 0.004 \\
\hline $\mathrm{C}_{5} \mathrm{H}_{8}$ & (Z)-1,3-Pentadiene & $1574-41-0$ & 68 & 8.68 & 0.005 \\
\hline $\mathrm{C}_{5} \mathrm{H}_{8}$ & 1,2-Pentadiene & $591-95-7$ & 68 & 8.94 & 0.002 \\
\hline $\mathrm{C}_{5} \mathrm{H}_{8}$ & 2,3-Pentadiene & $591-96-8$ & 68 & 9.03 & 0.001 \\
\hline $\mathrm{C}_{6} \mathrm{H}_{12}$ & 1-Hexene & $592-41-6$ & 84 & 11.54 & 0.041 \\
\hline $\mathrm{C}_{6} \mathrm{H}_{10}$ & 4-Methyl-1,3-pentadiene & $926-56-7$ & 82 & 12.66 & 0.020 \\
\hline $\mathrm{C}_{6} \mathrm{H}_{10}$ & 3-Hexyne & $928-49-4$ & 82 & 12.97 & 0.007 \\
\hline $\mathrm{C}_{6} \mathrm{H}_{10}$ & (Z)-3-Methyl-1,3-pentadiene & $2787-45-3$ & 82 & 13.22 & 0.006 \\
\hline $\mathrm{C}_{7} \mathrm{H}_{12}$ & 5-Methyl-1-hexyne & $2203-80-7$ & 96 & 13.92 & 0.001 \\
\hline $\mathrm{C}_{7} \mathrm{H}_{14}$ & 1-Heptene & $592-76-7$ & 98 & 15.52 & 0.025 \\
\hline $\mathrm{C}_{8} \mathrm{H}_{16}$ & 5-Methyl-3-heptene & $13172-91-3$ & 114 & 19.35 & 0.043 \\
\hline $\mathrm{C}_{8} \mathrm{H}_{16}$ & (E)-4-Octene & $14850-23-8$ & 112 & 19.52 & 0.022 \\
\hline
\end{tabular}




\begin{tabular}{|c|c|c|c|c|c|}
\hline & & & & & \\
\hline $\mathrm{C}_{8} \mathrm{H}_{16}$ & 2,3-Dimethyl-1-hexene & $16746-86-4$ & 112 & 19.73 & 0.038 \\
\hline $\mathrm{C}_{8} \mathrm{H}_{16}$ & (Z)-3-Octene & $14850-22-7$ & 112 & 19.63 & 0.038 \\
\hline $\mathrm{C}_{8} \mathrm{H}_{16}$ & (E)-2-Octene & $13389-42-9$ & 112 & 19.80 & 0.013 \\
\hline $\mathrm{C}_{9} \mathrm{H}_{18}$ & 1-Nonene & $124-11-8$ & 126 & 23.56 & 0.094 \\
\hline $\mathrm{C}_{10} \mathrm{H}_{20}$ & 1-Decene & $872-05-9$ & 140 & 27.17 & 0.012 \\
\hline $\mathrm{C}_{11} \mathrm{H}_{22}$ & 1-Undecene & $821-95-4$ & 154 & 30.53 & 0.009 \\
\hline $\mathrm{C}_{12} \mathrm{H}_{24}$ & 1-Dodecene & $112-41-4$ & 168 & 33.62 & 0.033 \\
\hline $\mathrm{C}_{13} \mathrm{H}_{26}$ & 1-Tridecene & $2437-56-1$ & 182 & 37.40 & 0.022 \\
\hline $\mathrm{C}_{14} \mathrm{H}_{28}$ & 1-Tetradecene & $1120-36-1$ & 196 & 43.03 & 0.026 \\
\hline $\mathrm{C}_{15} \mathrm{H}_{30}$ & 1-Pentadecene & $13360-61-7$ & 210 & 51.91 & 0.056 \\
\hline $\mathrm{C}_{16} \mathrm{H}_{32}$ & 1-Hexadecene & $629-73-2$ & 224 & 78.90 & 0.021 \\
\hline & & carbons & & & \\
\hline Cyclic alk & es and alkenes & & & & \\
\hline $\mathrm{C}_{6} \mathrm{H}_{10}$ & 4-Methylcyclopentene & $1759-81-5$ & 82 & 11.94 & 0.008 \\
\hline $\mathrm{C}_{8} \mathrm{H}_{16}$ & Pentylcyclopropane & $2511-91-3$ & 112 & 20.05 & 0.019 \\
\hline $\mathrm{C}_{8} \mathrm{H}_{14}$ & 3-Propylcyclopentene & $34067-75-9$ & 110 & 20.92 & 0.003 \\
\hline Arenes & & & & & \\
\hline $\mathrm{C}_{6} \mathrm{H}_{6}$ & Benzene & $71-43-2$ & 78 & 12.46 & 0.177 \\
\hline $\mathrm{C}_{7} \mathrm{H}_{8}$ & Toluene & $108-88-3$ & 92 & 16.87 & 0.084 \\
\hline $\mathrm{C}_{8} \mathrm{H}_{10}$ & Ethylbenzene & $100-41-4$ & 106 & 20.90 & 0.002 \\
\hline $\mathrm{C}_{8} \mathrm{H}_{10}$ & p-Xylene & $106-42-3$ & 106 & 21.17 & 0.109 \\
\hline $\mathrm{C}_{8} \mathrm{H}_{8}$ & Styrene & $100-42-5$ & 104 & 21.82 & 0.016 \\
\hline $\mathrm{C}_{9} \mathrm{H}_{12}$ & Propylbenzene & $103-65-1$ & 120 & 24.71 & 0.010 \\
\hline $\mathrm{C}_{10} \mathrm{H}_{14}$ & o-Cymene & $527-84-4$ & 134 & 27.54 & 0.020 \\
\hline $\mathrm{C}_{10} \mathrm{H}_{14}$ & Butylbenzene & $104-51-8$ & 134 & 28.48 & 0.013 \\
\hline $\mathrm{C}_{11} \mathrm{H}_{16}$ & Pentylbenzene & $538-68-1$ & 148 & 31.87 & 0.009 \\
\hline $\mathrm{C}_{12} \mathrm{H}_{18}$ & Hexylbenzene & $1077-16-3$ & 162 & 35.26 & 0.010 \\
\hline $\mathrm{C}_{13} \mathrm{H}_{20}$ & Heptylbenzene & $1078-71-3$ & 176 & 38.47 & 0.026 \\
\hline $\mathrm{C}_{14} \mathrm{H}_{22}$ & Octylbenzene & $2189-60-8$ & 190 & 45.06 & 0.008 \\
\hline $\mathrm{C}_{15} \mathrm{H}_{24}$ & Nonylbenzene & $1081-77-2$ & 204 & 60.30 & 0.029 \\
\hline Polycyclic & omatic hydrocarbons & & & & \\
\hline $\mathrm{C}_{10} \mathrm{H}_{8}$ & Naphthalene & $91-20-3$ & 128 & 32.07 & 0.018 \\
\hline $\mathrm{C}_{11} \mathrm{H}_{10}$ & 2-Methylnaphthalene & $91-57-6$ & 142 & 35.82 & 0.008 \\
\hline $\mathrm{C}_{11} \mathrm{H}_{10}$ & 1-Methylnaphthalene & $90-12-0$ & 142 & 36.34 & 0.009 \\
\hline & He & drocarbons & & & \\
\hline Dioxanes & & & & & \\
\hline $\mathrm{C}_{4} \mathrm{H}_{8} \mathrm{O}_{2}$ & 1,4-Dioxane & $123-91-1$ & 88 & 13.66 & 0.001 \\
\hline Furans & & & & & \\
\hline $\mathrm{C}_{4} \mathrm{H}_{4} \mathrm{O}$ & Furan & $110-00-9$ & 68 & 7.21 & 0.001 \\
\hline $\mathrm{C}_{5} \mathrm{H}_{6} \mathrm{O}$ & 2-Methylfuran & $534-22-5$ & 82 & 10.21 & 0.005 \\
\hline $\mathrm{C}_{5} \mathrm{H}_{6} \mathrm{O}$ & 3-Methylfuran & $930-27-8$ & 92 & 10.49 & 0.001 \\
\hline $\mathrm{C}_{6} \mathrm{H}_{8} \mathrm{O}$ & 2-Ethylfuran & $3208-16-0$ & 96 & 12.01 & 0.001 \\
\hline $\mathrm{C}_{7} \mathrm{H}_{10} \mathrm{O}$ & 2-Propylfuran & $4229-91-8$ & 110 & 18.34 & 0.002 \\
\hline $\mathrm{C}_{8} \mathrm{H}_{12} \mathrm{O}$ & 2-Butylfuran & $4466-24-4$ & 124 & 22.10 & 0.002 \\
\hline $\mathrm{C}_{9} \mathrm{H}_{14} \mathrm{O}$ & 2-Pentylfuran & $3777-69-3$ & 138 & 25.91 & 0.004 \\
\hline $\mathrm{C}_{10} \mathrm{H}_{16} \mathrm{O}$ & 2-Hexylfuran & $3777-70-6$ & 152 & 29.41 & 0.002 \\
\hline $\mathrm{C}_{11} \mathrm{H}_{18} \mathrm{O}$ & 2-Heptylfuran & $3777-71-7$ & 166 & 32.72 & 0.002 \\
\hline
\end{tabular}


\begin{tabular}{l|l}
$\mathrm{C}_{12} \mathrm{H}_{20} \mathrm{O}$ & 2-Octylfuran \\
$\mathrm{C}_{15} \mathrm{H}_{26} \mathrm{O}$ & 2-Decylfuran
\end{tabular}

Alcohols, ethers and esters

\begin{tabular}{l|l}
$\mathrm{CH}_{4} \mathrm{O}$ & Methanol \\
$\mathrm{C}_{2} \mathrm{H}_{6} \mathrm{O}$ & Ethanol \\
$\mathrm{C}_{4} \mathrm{H}_{10} \mathrm{O}$ & 1-Butanol \\
$\mathrm{C}_{5} \mathrm{H}_{8} \mathrm{O}_{2}$ & Methyl methacrylate \\
$\mathrm{C}_{6} \mathrm{H}_{6} \mathrm{O}$ & Phenol \\
$\mathrm{C}_{6} \mathrm{H}_{10} \mathrm{O}_{2}$ & $\gamma$-Caprolactone \\
$\mathrm{C}_{7} \mathrm{H}_{12} \mathrm{O}_{2}$ & $\gamma$-Heptanolactone \\
$\mathrm{C}_{8} \mathrm{H}_{14} \mathrm{O}_{2}$ & $\gamma$-Octalactone \\
$\mathrm{C}_{10} \mathrm{H}_{20} \mathrm{O}$ & $($ E)-2-Decen-1-ol \\
$\mathrm{C}_{9} \mathrm{H}_{16} \mathrm{O}_{2}$ & $\gamma$-Nonalactone \\
$\mathrm{C}_{10} \mathrm{H}_{18} \mathrm{O}_{2}$ & $\gamma$-Decalactone \\
$\mathrm{C}_{11} \mathrm{H}_{20} \mathrm{O}_{2}$ & $\gamma$-Undecalactone \\
$\mathrm{C}_{12} \mathrm{H}_{22} \mathrm{O}_{2}$ & $\gamma$-Dodecalactone \\
$\mathrm{C}_{13} \mathrm{H}_{24} \mathrm{O}_{2}$ & $\delta$-Tridecalactone
\end{tabular}

Aldehydes

$\mathrm{C}_{2} \mathrm{H}_{4} \mathrm{O}$

$\mathrm{C}_{3} \mathrm{H}_{4} \mathrm{O}$

$\mathrm{C}_{4} \mathrm{H}_{6} \mathrm{O}$

$\mathrm{C}_{4} \mathrm{H}_{8} \mathrm{O}$

$\mathrm{C}_{4} \mathrm{H}_{8} \mathrm{O}$

$\mathrm{C}_{5} \mathrm{H}_{10} \mathrm{O}$

$\mathrm{C}_{5} \mathrm{H}_{10} \mathrm{O}$

$\mathrm{C}_{5} \mathrm{H}_{4} \mathrm{O}_{2}$

$\mathrm{C}_{5} \mathrm{H}_{4} \mathrm{O}_{2}$

$\mathrm{C}_{6} \mathrm{H}_{12} \mathrm{O}$

$\mathrm{C}_{6} \mathrm{H}_{6} \mathrm{O}_{2}$

$\mathrm{C}_{7} \mathrm{H}_{14} \mathrm{O}$

$\mathrm{C}_{7} \mathrm{H}_{6} \mathrm{O}$

$\mathrm{C}_{8} \mathrm{H}_{16} \mathrm{O}$

$\mathrm{C}_{8} \mathrm{H}_{16} \mathrm{O}$

$\mathrm{C}_{9} \mathrm{H}_{18} \mathrm{O}$

$\mathrm{C}_{6} \mathrm{H}_{6} \mathrm{O}_{3}$

$\mathrm{C}_{10} \mathrm{H}_{20} \mathrm{O}$

$\mathrm{C}_{11} \mathrm{H}_{22} \mathrm{O}$

$\mathrm{C}_{12} \mathrm{H}_{24} \mathrm{O}$

$\mathrm{C}_{13} \mathrm{H}_{26} \mathrm{O}$

$\mathrm{C}_{14} \mathrm{H}_{28} \mathrm{O}$

Ketones

$\mathrm{C}_{3} \mathrm{H}_{6} \mathrm{O}$

$\mathrm{C}_{4} \mathrm{H}_{6} \mathrm{O}_{2}$

$\mathrm{C}_{4} \mathrm{H}_{8} \mathrm{O}$

$\mathrm{C}_{5} \mathrm{H}_{10} \mathrm{O}$

$\mathrm{C}_{5} \mathrm{H}_{8} \mathrm{O}$

$\mathrm{C}_{6} \mathrm{H}_{12} \mathrm{O}$

Acetaldehyde

2-Propenal

2-Methyl-2-propenal

2-Methylpropanal

n-Butanal

3-Methylbutanal

n-Pentanal

Furfural

3-Furaldehyde

n-Hexanal

n-Heptanal

Benzaldehyde

2-Ethylhexanal

n-Octanal

n-Nonanal

n-Decanal

n-Undecanal

n-Dodecanal

n-Tridecanal

n-Tetradecanal

Cyclopentanone

2-Hexanone \begin{tabular}{|l|}
$4179-38-8$ \\
$83469-85-6$
\end{tabular}

180
222

36.35

69.21

0.002

\section{Oxygenated hydrocarbons}

\begin{tabular}{|l|c|c|c|}
$67-56-1$ & 32 & 4.61 & 0.237 \\
$64-17-5$ & 46 & 6.63 & 0.114 \\
$71-36-3$ & 74 & 12.54 & 0.012 \\
$80-62-6$ & 100 & 14.39 & 0.017 \\
$108-95-2$ & 94 & 24.78 & 0.015 \\
$695-06-7$ & 114 & 27.01 & 0.002 \\
$105-21-5$ & 128 & 31.11 & 0.006 \\
$104-50-7$ & 142 & 34.56 & 0.008 \\
$18409-18-2$ & 156 & 36.50 & 0.053 \\
$104-61-0$ & 156 & 39.02 & 0.009 \\
$706-14-9$ & 170 & 45.76 & 0.009 \\
$104-67-6$ & 184 & 52.97 & 0.003 \\
$2305-05-7$ & 198 & 72.91 & 0.010 \\
$7370-92-5$ & 212 & 106.73 & 0.002
\end{tabular}

5.33

7.29

9.69

9.73

10.53

13.64

14.65

17.47

18.35

18.94

23.07

23.07

24.06

25.78

26.78

30.36

30.91

33.22

37.02

42.00

50.93

64.18

7.68

10.59

10.71

14.42

16.90

18.69
5-Methyl-2-furancarboxaldehyde

5-Hydroxymethylfurfural

75-07-0

$107-02-8$

$78-85-3$

$78-84-2$

123-72-8

590-86-3

110-62-3

98-01-1

498-60-2

66-25-1

620-02-0

111-71-7

100-52-7

123-05-7

124-13-0

124-19-6

67-47-0

112-31-2

112-44-7

112-54-9

10486-19-8

$124-25-4$

67-64-1

431-03-8

78-93-3

107-87-9

120-92-3

591-78-6

\begin{tabular}{c|}
44 \\
56 \\
70 \\
72 \\
72 \\
86 \\
86 \\
96 \\
96 \\
100 \\
110 \\
114 \\
106 \\
128 \\
128 \\
142 \\
126 \\
156 \\
170 \\
184 \\
198 \\
212
\end{tabular}

0.276

0.005

0.019

0.021

0.005

0.016

0.022

0.001

0.035

0.072

0.002

0.101

0.038

0.024

0.026

0.006

0.004

0.050

0.014

0.042

0.008

0.044

0.341

0.001

0.013

0.016 


\begin{tabular}{|c|c|c|c|c|c|}
\hline & & & & & \\
\hline $\mathrm{C}_{7} \mathrm{H}_{14} \mathrm{O}$ & 2-Heptanone & $110-43-0$ & 114 & 22.77 & 0.020 \\
\hline $\mathrm{C}_{8} \mathrm{H}_{14} \mathrm{O}$ & 6-Methyl-5-hepten-2-one & $110-93-0$ & 126 & 26.00 & 0.016 \\
\hline $\mathrm{C}_{8} \mathrm{H}_{16} \mathrm{O}$ & 2-Octanone & $111-13-7$ & 128 & 26.53 & 0.019 \\
\hline $\mathrm{C}_{9} \mathrm{H}_{18} \mathrm{O}$ & 2-Nonanone & $821-55-6$ & 142 & 30.01 & 0.034 \\
\hline $\mathrm{C}_{10} \mathrm{H}_{20} \mathrm{O}$ & 2-Decanone & $693-54-9$ & 156 & 32.89 & 0.031 \\
\hline $\mathrm{C}_{8} \mathrm{H}_{4} \mathrm{O}_{3}$ & Phthalic anhydride & $85-44-9$ & 148 & 36.09 & 0.092 \\
\hline $\mathrm{C}_{11} \mathrm{H}_{22} \mathrm{O}$ & 2-Undecanone & $112-12-9$ & 170 & 36.85 & 0.019 \\
\hline $\mathrm{C}_{12} \mathrm{H}_{24} \mathrm{O}$ & 2-Dodecanone & $6175-49-1$ & 184 & 41.55 & 0.049 \\
\hline $\mathrm{C}_{13} \mathrm{H}_{26} \mathrm{O}$ & 2-Tridecanone & $593-08-8$ & 198 & 50.73 & 0.013 \\
\hline $\mathrm{C}_{14} \mathrm{H}_{28} \mathrm{O}$ & 2-Tetradecanone & $2345-27-9$ & 212 & 60.90 & 0.028 \\
\hline $\mathrm{C}_{15} \mathrm{H}_{30} \mathrm{O}$ & 2-Pentadecanone & $2345-28-0$ & 226 & 82.85 & 0.027 \\
\hline Carboxylic & cids & & & & \\
\hline $\mathrm{C}_{2} \mathrm{H}_{4} \mathrm{O}_{2}$ & Acetic acid & $64-19-7$ & 60 & 11.31 & 0.131 \\
\hline $\mathrm{C}_{3} \mathrm{H}_{6} \mathrm{O}_{2}$ & n-Propanoic acid & $79-09-4$ & 74 & 15.37 & 0.014 \\
\hline $\mathrm{C}_{4} \mathrm{H}_{8} \mathrm{O}_{2}$ & n-Butanoic acid & $107-92-6$ & 88 & 19.04 & 0.050 \\
\hline $\mathrm{C}_{5} \mathrm{H}_{10} \mathrm{O}_{2}$ & 3-Methylbutanoic acid & $503-74-2$ & 102 & 22.10 & 0.009 \\
\hline $\mathrm{C}_{5} \mathrm{H}_{10} \mathrm{O}_{2}$ & n-Pentanoic acid & $109-52-4$ & 102 & 23.02 & 0.035 \\
\hline $\mathrm{C}_{6} \mathrm{H}_{12} \mathrm{O}_{2}$ & n-Hexanoic acid & $142-62-1$ & 116 & 26.51 & 0.072 \\
\hline $\mathrm{C}_{7} \mathrm{H}_{14} \mathrm{O}_{2}$ & n-Heptanoic acid & $111-14-8$ & 130 & 29.93 & 0.031 \\
\hline $\mathrm{C}_{8} \mathrm{H}_{16} \mathrm{O}_{2}$ & n-Octanoic acid & $124-07-2$ & 144 & 33.01 & 0.075 \\
\hline $\mathrm{C}_{9} \mathrm{H}_{18} \mathrm{O}_{2}$ & n-Nonanoic acid & $112-05-0$ & 158 & 36.49 & 0.047 \\
\hline $\mathrm{C}_{10} \mathrm{H}_{20} \mathrm{O}_{2}$ & n-Decanoic acid & $334-48-5$ & 172 & 41.60 & 0.096 \\
\hline $\mathrm{C}_{11} \mathrm{H}_{22} \mathrm{O}_{2}$ & n-Undecanoic acid & $112-37-8$ & 186 & 49.76 & 0.037 \\
\hline $\mathrm{C}_{12} \mathrm{H}_{24} \mathrm{O}_{2}$ & n-Dodecanoic acid & $143-07-7$ & 200 & 62.22 & 0.075 \\
\hline & Sul & mpounds & & & \\
\hline $\mathrm{H}_{2} \mathrm{~S}$ & Hydrogen sulfide & $7783-06-4$ & 34 & 2.83 & 0.042 \\
\hline $\operatorname{COS}$ & Carbonyl sulfide & $463-58-1$ & 60 & 3.50 & 0.073 \\
\hline $\mathrm{O}_{2} \mathrm{~S}$ & Sulfur dioxide & $7446-09-5$ & 64 & 4.76 & 0.218 \\
\hline $\mathrm{CH}_{4} \mathrm{~S}$ & Methanethiol & $74-93-1$ & 48 & 5.51 & 0.093 \\
\hline $\mathrm{CS}_{2}$ & Carbon disulfide & $75-15-0$ & 76 & 7.79 & 0.025 \\
\hline $\mathrm{C}_{2} \mathrm{H}_{6} \mathrm{~S}$ & Dimethyl sulfide & $75-18-3$ & 62 & 7.76 & 1.585 \\
\hline $\mathrm{C}_{2} \mathrm{H}_{6} \mathrm{~S}_{2}$ & Dimethyl disulfide & $624-92-0$ & 94 & 15.27 & 0.005 \\
\hline $\mathrm{C}_{10} \mathrm{H}_{16} \mathrm{~S}$ & 2-Hexylthiophene & $18794-77-9$ & 168 & 35.04 & 0.002 \\
\hline & Nitro & ompounds & & & \\
\hline $\mathrm{N}_{2}$ & Nitrogen & $7727-37-9$ & 28 & 1.68 & 1.057 \\
\hline $\mathrm{H}_{3} \mathrm{~N}$ & Ammonia & $7664-41-7$ & 17 & 3.01 & 0.046 \\
\hline $\mathrm{C}_{2} \mathrm{H}_{3} \mathrm{~N}$ & 2H-Azirine & $157-16-4$ & 41 & 6.93 & 0.394 \\
\hline $\mathrm{CH}_{3} \mathrm{NO}$ & Formamide & $75-12-7$ & 45 & 7.76 & 0.436 \\
\hline $\mathrm{C}_{3} \mathrm{H}_{5} \mathrm{~N}$ & Propanenitrile & $107-12-0$ & 55 & 9.53 & 0.030 \\
\hline $\mathrm{C}_{4} \mathrm{H}_{5} \mathrm{~N}$ & Pyrrole & $109-97-7$ & 67 & 14.57 & 0.004 \\
\hline $\mathrm{C}_{5} \mathrm{H}_{5} \mathrm{~N}$ & Pyridine & $110-86-1$ & 79 & 15.75 & 0.020 \\
\hline $\mathrm{C}_{5} \mathrm{H}_{9} \mathrm{~N}$ & Pentanenitrile & $110-59-8$ & 83 & 17.55 & 0.002 \\
\hline $\mathrm{C}_{6} \mathrm{H}_{9} \mathrm{~N}$ & 2,3-Dimethyl-1H-pyrrole & $600-28-2$ & 95 & 18.85 & 0.001 \\
\hline $\mathrm{C}_{6} \mathrm{H}_{11} \mathrm{NO}$ & 6-Methyl-2-piperidinone & $4775-98-8$ & 113 & 21.73 & 0.002 \\
\hline $\mathrm{C}_{7} \mathrm{H}_{5} \mathrm{~N}$ & Benzonitrile & $100-47-0$ & 103 & 24.88 & 0.094 \\
\hline $\mathrm{C}_{7} \mathrm{H}_{13} \mathrm{~N}$ & Heptanonitrile & $629-08-3$ & 111 & 25.76 & 0.012 \\
\hline $\mathrm{C}_{4} \mathrm{H}_{5} \mathrm{NO}_{2}$ & Succinimide & $123-56-8$ & 99 & 29.04 & 0.008 \\
\hline $\mathrm{C}_{8} \mathrm{H}_{5} \mathrm{NO}_{2}$ & Phthalimide & $85-41-6$ & 147 & 43.66 & 0.013 \\
\hline
\end{tabular}


$\mathrm{C}_{9} \mathrm{H}_{11} \mathrm{NO} \quad$ Phenylpropanamide

$\mid 102-93-2$
Inorganic compounds

Oxides

\begin{tabular}{l|l|l|l|l|l}
$\mathrm{H}_{2} \mathrm{O}$ & Water & $7732-18-5$ & 18 & 3.26 & 17.953 \\
$\mathrm{CO}_{2}$ & Carbon dioxide & $124-38-9$ & 44 & 1.92 & 72.462 \\
\hline
\end{tabular}

Note: ${ }^{1}$ CAS - unique numerical identifier of chemical compounds included in the register Chemical Abstracts Service (https://www.cas.org); ${ }^{2} \mathrm{MW}$ - nominal mass; ${ }^{3} \mathrm{RT}$ - retention time; ${ }^{4} \mathrm{~A}$ - normalized area (the area ratio of the individual gas mixture components to the summ of the areas of all the components in the chromatogram). 